\title{
Light hadron masses from a matrix model for QCD
}

\author{
Mahul Pandey ${ }^{1}$ and Sachindeo Vaidya $\odot^{2}$ \\ ${ }^{1}$ School of Theoretical Physics, Dublin Institute for Advanced Studies, Dublin 4 D04 C932, Ireland \\ ${ }^{2}$ Centre for High Energy Physics, Indian Institute of Science, Bengaluru 560012, India
}

(Received 3 January 2020; accepted 2 June 2020; published 22 June 2020)

\begin{abstract}
The $S U(3)$ Yang-Mills matrix model coupled to fundamental fermions is an approximation of quantum chromodynamics (QCD) on a 3-sphere of radius $R$. The spectrum of this matrix model Hamiltonian is estimated using standard variational methods, and is analyzed in the strong coupling limit. By employing a matching prescription to determine the dependence of the Yang-Mills coupling constant $g$ on $R$, we relate the asymptotic values of the energy eigenvalues in the $R \rightarrow \infty$ (flat space) limit to the masses of light hadrons. We find that the matrix model estimates the light hadron spectrum fairly accurately, with the light baryon masses falling within $10 \%$, and most light meson masses falling within about $30 \%$ of their observed values.
\end{abstract}

DOI: 10.1103/PhysRevD.101.114020

\section{INTRODUCTION}

Hadrons constitute almost all of ordinary matter, and the study of their properties is of fundamental importance to test the predictions of low-energy quantum chromodynamics (QCD). However, the fact that hadrons lie deep in the nonperturbative regime of the theory poses a considerable challenge for theoretical physicists to predict their properties reliably. Much of our knowledge in this regime comes from numerical simulations in lattice QCD, latest results including [1-16] for light hadron spectrum and [17-25] for charmed hadrons.

A simple and elegant matrix model of Yang-Mills theory, capable of capturing important topological features of the full quantum field theory, was obtained in [26,27]. The matrix model corresponds to a dimensional reduction of Yang-Mills theory on $S^{3} \times \mathbb{R}$, and is a quantum-mechanical model based on $3 \times\left(N^{2}-1\right)$-dimensional real matrices as degrees of freedom. This model proves to be quite good in describing the mass spectrum of glueballs [28] in the low energy regime of pure Yang-Mills theory.

In this paper, we push the $S U(3)$ matrix model further by coupling fundamental quarks and provide variational estimates for the masses of light hadrons. Specifically, we give estimates for the masses of the light pseudoscalar and vector mesons and spin- $\frac{1}{2}$ and spin- $-\frac{3}{2}$ baryons, which turn out to be surprisingly successful, as can be seen from Fig. 5 and Tables IV and V.

Published by the American Physical Society under the terms of the Creative Commons Attribution 4.0 International license. Further distribution of this work must maintain attribution to the author(s) and the published article's title, journal citation, and DOI. Funded by SCOAP.
We consider this model as a new proposal for describing the extreme low energy sector of QCD, i.e., the sector describing light hadrons. Just as in QCD, it has exactly four bare parameters, namely, the masses $m_{u}, m_{d}$ and $m_{s}$ of the up, down and strange quark, the Yang-Mills coupling $g$. The radius $R$ of the 3 -sphere simply sets the overall scale in the model. To relate the energy spectrum of the matrix model to physical masses of particles, we need to employ a suitable matching scheme, detailed later in the paper, which involves using the observed masses of four hadrons as inputs. Once the matching scheme has been used to fix the unknown parameters and to set the overall scale, the masses of the other particles can be unambiguously determined from the matrix model.

Although this use of the matrix model in hadron spectroscopy is new, the model itself has appeared earlier in other contexts. For instance, Myers has studied this model to examine the dynamics of $N$ D0-branes in a specific external Ramond-Ramond field [29]. The energy spectrum of this model, however, has not been investigated thoroughly. We do so here using traditional and wellestablished variational methods. We find it striking that such a simple model, analyzed using such modest human and computational resources, is able to provide estimates of light hadrons masses with such accuracy.

The paper is organized as follows. In Sec. II, we briefly review the pure gauge matrix model of [26,27] and describe the inclusion of quarks. In Sec. III, we review the variational method for energy eigenvalue determination. In Sec. IV, we set up the mass-matching scheme. Our results are given in Sec. V, and a discussion of the results in Sec. VI.

\section{THE MATRIX MODEL WITH QUARKS}

The matrix model $[26,27]$ for pure Yang-Mills theory is constructed by isomorphically mapping the spatial $S^{3}$ to an 
$S U$ (2) subgroup of the gauge group $S U(N)$, and the pulling back of the general left-invariant one-form on $S U(N)$ under this map. The general left-invariant one-form on $S U(N)$ can be expressed as $\Omega=\operatorname{Tr}\left(T_{a} g^{-1} d g\right) M_{a b} T_{b}$ where $g \in S U(N), T_{a}$ are the generators in fundamental representation, and $M$ is a $\left(N^{2}-1\right)$-dimensional real matrix. Under the isomorphic mapping, the spatial vector fields are identified with $i X_{i}$, where $X_{i}$ are the left-invariant vector fields in the Lie algebra of $S U(2)$. The pullback of $\Omega$ under this map gives the gauge fields as $A_{0}=0$, $A_{i}=M_{i a} T_{a}$. The matrices $M_{i a}$ are $3 \times\left(N^{2}-1\right)$ rectangular matrices, and represent our gauge variables.

The curvature $F_{i j}$ corresponding to $A_{i}$ is obtained by the pullback of the curvature associated with $\Omega$ to the spatial $S^{3}$ :

$$
\begin{aligned}
F_{i j} & =(d \Omega+\Omega \wedge \Omega)\left(X_{i}, X_{j}\right) \\
& =\left(-\frac{1}{R} \epsilon_{i j k} M_{k a}+f_{a b c} M_{i b} M_{k c}\right) T_{a} .
\end{aligned}
$$

Then the chromoelectric and chromomagnetic fields read

$$
\begin{aligned}
E_{i a} & \equiv F_{0 i a}=\dot{M}_{i a}, \\
B_{i a} & \equiv \frac{1}{2} \epsilon_{i j k} F_{j k a}=-\frac{1}{R} M_{i a}+\frac{1}{2} \epsilon_{i j k} f_{a b c} M_{j b} M_{k c} .
\end{aligned}
$$

We ignore the numerical factor in the volume of $S^{3}$ and take it to be $R^{3}$. The matrix model Lagrangian is

$$
L_{\mathrm{YM}}=-\frac{R^{3}}{4 g^{2}} F_{\mu \nu}^{a} F^{a \mu \nu}=\frac{R^{3}}{2 g^{2}}\left(E_{i}^{a} E_{i}^{a}-B_{i}^{a} B_{i}^{a}\right) .
$$

In the temporal gauge $A_{0}=0$, the Lagrangian is expanded to obtain quadratic, cubic and quartic interactions in the potential:

$$
\begin{aligned}
L_{\mathrm{YM}}= & \frac{R^{3}}{2 g^{2}}\left(\dot{M}_{i a} \dot{M}_{i a}-\frac{1}{R^{2}} M_{i a} M_{i a}+\frac{1}{R} \epsilon_{i j k} f_{a b c} M_{i a} M_{j b} M_{k c}\right. \\
& \left.-\frac{1}{2} f_{a b c} f_{a d e} M_{i b} M_{j c} M_{i d} M_{j e}\right) .
\end{aligned}
$$

To the matrix model Lagrangian (2.5), we add minimally coupled massive 3-flavor quarks $Q$ in the Dirac representation, transforming in the fundamental representation of color $S U(3)$, and flavor $S U(3)$. The spinor $Q \equiv\left(Q_{f \alpha A}\right)$ carries indices $f, \alpha$ and $A$ denoting flavor, spin and color indices respectively. Under gauge transformations $u \in S U(3)$, spatial rotations $R \in S O(3)$ and flavor rotations $v \in S U(3), Q_{f \alpha A}$ transforms as

$$
\begin{aligned}
Q_{f \alpha A} & \rightarrow u_{A B} Q_{f \alpha B}, \quad A, B=1,2,3, \\
Q_{f \alpha A} & \rightarrow D^{\frac{1}{2}}(R)_{\alpha \beta} Q_{f \beta A}, \quad \alpha, \beta=1,2, \\
Q_{f \alpha A} & \rightarrow v_{f g} Q_{g \alpha A}, \quad f, g=1,2,3
\end{aligned}
$$

where $D^{\frac{1}{2}}(R)_{\alpha \beta}$ is the spin- $\frac{1}{2}$ representation of $R$.
A Dirac fermion $Q$ is made up of a left Weyl fermion $b$ and a right Weyl fermion $c^{\dagger}$ :

$$
Q_{f \alpha A}=\left(\begin{array}{c}
b_{f \alpha A} \\
-i\left(\sigma_{2}\right)_{\alpha \beta} c_{f \beta A}^{\dagger}
\end{array}\right) \equiv\left(\begin{array}{c}
b_{f \alpha A} \\
d_{f \alpha A}^{\dagger}
\end{array}\right) \text {. }
$$

This implies that $b$ transforms in the fundamental and $d$ in the antifundamental representations of color and flavor, and both have the same transformation property under rotations:

$$
\begin{aligned}
& b_{f \alpha A} \rightarrow u_{A B} b_{f \alpha B}, \quad A, B=1,2,3, \\
& b_{f \alpha A} \rightarrow D^{\frac{1}{2}}(R)_{\alpha \beta} b_{f \beta A}, \quad \alpha, \beta=1,2, \\
& b_{f \alpha A} \rightarrow v_{f g} b_{g \alpha A}, \quad f, g=1,2,3
\end{aligned}
$$

and

$$
\begin{aligned}
& d_{f \alpha A} \rightarrow u_{A B}^{*} d_{f \alpha B}, \quad A, B=1,2,3, \\
& d_{f \alpha A} \rightarrow D^{\frac{1}{2}}(R)_{\alpha \beta} d_{f \beta A}, \quad \alpha, \beta=1,2, \\
& d_{f \alpha A} \rightarrow v_{f g}^{*} d_{g \alpha A}, \quad f, g=1,2,3 .
\end{aligned}
$$

The Lagrangian for minimally coupled massive quarks on a sphere is given by [30]

$$
\begin{aligned}
L= & -\frac{R^{3}}{4 g^{2}} F_{\mu \nu}^{a} F^{a \mu \nu} \\
& +R^{3}\left(i \bar{Q}_{f} \gamma^{\mu}\left(\mathcal{D}_{\mu} Q_{f}\right)-m_{f} \bar{Q}_{f} Q_{f}-\frac{3}{2 R} \bar{Q}_{f} \gamma^{5} \gamma^{0} Q_{f}\right)
\end{aligned}
$$

where $\bar{Q}=Q^{\dagger} \gamma^{0}$. The last term in Eq. (2.16) comes from the nonzero curvature of the spatial $S^{3}$.

Both the gauge fields $M_{i a}$ and the quarks $b$ and $d$ depend only on time. $M$ has dimensions of inverse length. To express the Hamiltonian in terms of dimensionless quantities and for computational ease, it is convenient to rescale $M \rightarrow \frac{g M}{R}$. Also, to make the fermionic variables dimensionless, we rescale $b \rightarrow R^{-\frac{3}{2}} b, d \rightarrow R^{-\frac{3}{2}} d$, and express the quark masses in units of $R^{-1}, m_{f} \equiv \frac{\mu_{f}}{R}$. The Hamiltonian then works out to be

$$
H=\frac{1}{R}\left(H_{\mathrm{YM}}+H_{m}+H_{c}+H_{\mathrm{int}}\right)
$$

where 


$$
\begin{aligned}
H_{\mathrm{YM}}= & \frac{1}{2} \Pi_{i a} \Pi_{i a}+\frac{1}{2} M_{i a} M_{i a}-\frac{g}{2} \epsilon_{i j k} f_{a b c} M_{i a} M_{j b} M_{k c} \\
& +\frac{g^{2}}{4} f_{a b c} f_{a d e} M_{i b} M_{j c} M_{i d} M_{j e}, \\
H_{m}= & \sum_{f} \mu_{f}\left(b_{f \alpha A}^{\dagger} b_{f \alpha A}-d_{f \alpha A} d_{f \alpha A}^{\dagger}\right), \\
H_{c}= & \frac{3}{2}\left(b_{f \alpha A}^{\dagger} d_{f \alpha A}^{\dagger}+d_{f \alpha A} b_{f \alpha A}\right), \\
H_{\mathrm{int}}= & -\frac{g}{2} M_{i a}\left(b_{f \alpha A}^{\dagger} \sigma_{\alpha \beta}^{i} \lambda_{A B}^{a} d_{f \beta B}^{\dagger}+d_{f \alpha A} \sigma_{\alpha \beta}^{i} \lambda_{A B}^{a} b_{f \beta B}\right) .
\end{aligned}
$$

Here $\lambda^{a}$ are the Gell-Mann matrices.

The Gauss's law constraint is

$$
G_{a}=f_{a b c} \Pi_{i b} M_{i c}-\frac{1}{2}\left(b_{f}^{\dagger} \lambda_{a} b_{f}-d_{f} \lambda_{a} d_{f}^{\dagger}\right),
$$

where the suppressed indices are understood as being summed over.

To quantize the system, we impose the canonical commutation (and anticommutation) relations

$$
\begin{aligned}
{\left[M_{i a}, \Pi_{j b}\right] } & =i \delta_{i j} \delta_{a b}, \\
\left\{b_{f \alpha A}, b_{g \beta B}^{\dagger}\right\} & =\delta_{\alpha \beta} \delta_{A B} \delta_{f g}, \\
\left\{d_{f \alpha A}, d_{g \beta B}^{\dagger}\right\} & =\delta_{\alpha \beta} \delta_{A B} \delta_{f g}
\end{aligned}
$$

and demand that all physical states $|\Psi\rangle_{\text {phys }}$ be annihilated by the Gauss law:

$$
G_{a}|\Psi\rangle_{\text {phys }}=0 .
$$

Total spin, given by

$$
J_{i}=\epsilon_{i j k} M_{j a} \Pi_{k a}+\frac{1}{2}\left(b_{f}^{\dagger} \sigma_{i} b_{f}-d_{f} \sigma_{i} d_{f}^{\dagger}\right),
$$

commutes with $H$ and so its eigenstates can be assigned a spin unambiguously.

In the fundamental representation, the generators of flavor symmetry are given by $\mathcal{T}_{F} \equiv \frac{1}{2} \lambda_{F}, F=1, \ldots, 8$, $\lambda_{F}$ being the Gell-Mann matrices. In terms of these generators, we can define the isospin operator $I_{3}$ and the hypercharge $Y$ as

$$
\begin{aligned}
& I_{3}=Q^{\dagger} \mathcal{T}_{3} Q=b^{\dagger} \mathcal{T}_{3} b+d \mathcal{T}_{3} d^{\dagger}, \\
& Y=\frac{2}{\sqrt{3}} Q^{\dagger} \mathcal{T}_{8} Q=\frac{2}{\sqrt{3}}\left(b^{\dagger} \mathcal{T}_{8} b+d \mathcal{T}_{8} d^{\dagger}\right) .
\end{aligned}
$$

These operators also commute with $H$ and so the eigenstates can be assigned isospin and hypercharge.
We will also need the fermion number operator

$$
N_{Q}=b^{\dagger} b+d^{\dagger} d .
$$

Eigenstates of $N_{Q}$ have a definite number of quarks + antiquarks.

To estimate light hadron masses, we choose a set of variational states $\left\{\left|\Psi_{n}\right\rangle\right\}$ and organize them according to their spin and flavor quantum numbers. Then, we compute $\tilde{H}_{m n} \equiv\left\langle\Psi_{m}|H| \Psi_{n}\right\rangle$ and the Gram matrix $S_{m n}=\left\langle\Psi_{m} \mid \Psi_{n}\right\rangle$, defined in Sec. III, and evaluate the generalized eigenvalues and eigenvectors of $\tilde{H}$ with respect to $S$. The eigenvalues have functional dependence on the bare parameters of the theory, namely, the quark masses $\mu_{f}$ and the coupling constant $g$, as well as the infrared cutoff $R$. To obtain welldefined values for light hadron masses, we need to prescribe a suitable renormalization scheme to determine the flow of the bare parameters with the scale $R$. A brief description of this renormalization procedure is given in Sec. IV.

\section{VARIATIONAL CALCULATION}

To estimate the eigenvalue spectrum of the matrix model Hamiltonian, we use the Rayleigh-Ritz variational method. We briefly summarize the steps for the variational calculation in the following paragraphs.

We first construct a trial set of variational states $\left\{\left|\psi_{i}\right\rangle\right\}$. Then we consider the following variational ansatz:

$$
|\chi\rangle=\sum_{i} c_{i}\left|\psi_{i}\right\rangle, \quad c_{i} \in \mathbb{C} .
$$

To estimate the spectrum, consider the functional

$$
\mathcal{K}=\langle\chi|H| \chi\rangle-\lambda(\langle\chi \mid \chi\rangle-1)
$$

where $\lambda$ is a Lagrange multiplier for the constraint that the state $|\chi\rangle$ is normalized to 1 .

Minimizing this functional with respect to $c_{i}^{*}$ leads to the generalized eigenvalue equation,

$$
\sum_{j} \tilde{H}_{i j} c_{j}=\lambda \sum_{j} \tilde{S}_{i j} c_{j},
$$

where $\tilde{H}_{i j} \equiv\left\langle\psi_{i}|H| \psi_{j}\right\rangle$ and $S_{i j} \equiv\left\langle\psi_{i} \mid \psi_{j}\right\rangle$.

Minimizing with respect to $\lambda$ yields

$$
\sum_{i j} c_{i}^{*} S_{i j} c_{j}=0
$$

Equations (3.3) and (3.4) together give

$$
\lambda=\frac{\sum_{j k} c_{k}^{*} \tilde{H}_{k j} c_{j}}{\sum_{j k} c_{k}^{*} S_{k j} c_{j}}=\frac{\langle\chi|H| \chi\rangle}{\langle\chi \mid \chi\rangle} .
$$


Thus, the lowest eigenvalue of the matrix $\tilde{H}$ with respect to $S$ gives the ground state energy and the higher eigenvalues give the energies of the excited states. Furthermore, the variational states can be organized via their quantum numbers under observables that commute with $H$, for example total spin, isospin and hypercharge. $\tilde{H}$ then assumes a block-diagonal form, and each block can be treated separately.

A typical variational state in our ansatz is a combination of a fermionic and a bosonic wave function. The fermionic part of the wave function can be created by successive operation of the fermion and antifermion creation operators $b^{\dagger}$ and $d^{\dagger}$ respectively on the fermion Fock vacuum, denoted by $\left|0_{F}\right\rangle$. As the bosonic part of the wave function, we take eigenfunctions of the 24-dimensional bosonic oscillator, given by

$$
H_{\mathrm{osc}} \equiv \frac{1}{2}\left(\Pi_{i a} \Pi_{i a}+M_{i a} M_{i a}\right) .
$$

To construct our variational ansatz, we take combinations of different bosonic and fermionic wave functions such that the combination as a whole transforms as a color singlet. In this article, we consider fermionic states with up to five fermions, and bosonic states with up to four bosonic oscillators. They are combined in different ways to give 449 variational states in the ansatz for scalar mesons, 1017 states for vector mesons, 449 states for the baryon octet and 604 states for the baryon decuplet.

The procedure for constructing the variational states is outlined briefly in the Appendix, where we also list all linearly independent fermionic and bosonic wave functions in our ansatz.

\section{LIGHT HADRONS FROM THE SPECTRUM OF $H$}

The energy eigenvalues of $H$ in general have functional dependence on four parameters in our theory, namely, the quark masses $\mu_{f}, f=1, \ldots, 3$ and the coupling constant $g$. As an approximation, we take the bare masses of the up and down quarks to be the same. Thus, we have two mass parameters, $\mu \equiv \mu_{u / d}$ and $\mu_{s}$, and the coupling constant $g$. We also have a length scale in our theory, which is the infrared cutoff $R$. The bare parameters $\mu, \mu_{s}$ and $g$ have no physical meaning. To obtain meaningful results in the flat space, or $R \rightarrow \infty$ limit, the bare parameters are chosen to depend on $R$ in such a way that the energy eigenvalues asymptote to fixed numbers at large $R$.

The model takes into account only the zero mode sector of the full quantum field theory. The (renormalized) zeropoint energy of the higher momentum modes of the full quantum field theory can at most change the spectrum of our Hamiltonian by an overall additive constant $C(R)$. Energy differences, of course, will not depend on $C(R)$.
Energy eigenvalues of (2.17), taking into account the shift $C(R)$, have the functional form

$$
\mathcal{E}_{n}^{k}\left(\mu, \mu_{s}, g, R\right) \equiv \frac{f_{n}^{k}\left(\mu, \mu_{s}, g\right)}{R}+C(R)
$$

where $k$ denotes the quantum numbers (spin, isospin etc.) assigned to the corresponding eigenstates, and $n$ labels all the eigenstates with the same quantum numbers.

Let us look at the eigenvalues corresponding to four particles labeled by $\left(k_{i}, n_{i}\right), i=1, \ldots, 4$. We can define two ratios of the energy differences, which has functional dependence only on $\mu, \mu_{s}, g$ :

$$
\begin{gathered}
\mathcal{R}_{123}\left(\mu, \mu_{s}, g\right) \equiv \frac{f_{n_{1}}^{k_{1}}-f_{n_{2}}^{k_{2}}}{f_{n_{1}}^{k_{1}}-f_{n_{3}}^{k_{3}}}, \\
\mathcal{R}_{423}\left(\mu, \mu_{s}, g\right) \equiv \frac{f_{n_{4}}^{k_{4}}-f_{n_{2}}^{k_{2}}}{f_{n_{1}}^{k_{1}}-f_{n_{3}}^{k_{3}}} .
\end{gathered}
$$

We now demand that the two ratios be fixed to their observed values for all $g$. In other words, we look for solutions to the set of equations,

$\mathcal{R}_{123}\left(\mu, \mu_{s}, g\right)=R_{123}^{\mathrm{obs}}, \quad \mathcal{R}_{423}\left(\mu, \mu_{s}, g\right)=R_{423}^{\mathrm{obs}}$,

where $R^{\text {obs }}$ denotes the observed values of the two ratios. Solving (4.4) gives us two functions $\mu(g)$ and $\mu_{s}(g)$. With this choice of $\mu(g)$ and $\mu_{s}(g)$, the ratio of the energy differences between any two pairs of particles can be computed. In particular, let us define

$$
\mathcal{R}_{x}(g) \equiv \frac{f_{n_{x}}^{k_{x}}-f_{n_{2}}^{k_{2}}}{f_{n_{1}}^{k_{1}}-f_{n_{3}}^{k_{3}}}
$$

where $x \neq 1,2,3,4$. We observe that for each $x, \mathcal{R}_{x}(g)$ approaches a well-defined asymptotic value for large $g$. These ratios are predictions of our model and listed in Table III.

Let us now see how to determine the mass differences themselves. To this end, let us look at a particular energy difference, say between particles 1 and 2, and substitute the functions $\mu(g)$ and $\mu_{s}(g)$ in its expression:

$$
\begin{aligned}
\Delta \mathcal{E}_{12}(g, R) & \equiv \mathcal{E}_{n_{1}}^{k_{1}}-\mathcal{E}_{n_{2}}^{k_{2}} \\
& =\frac{f_{n_{1}}^{k_{1}}\left(g, \mu(g), \mu_{s}(g)\right)-f_{n_{2}}^{k_{2}}\left(g, \mu(g), \mu_{s}(g)\right)}{R} .
\end{aligned}
$$

We again demand that for all $R, \Delta \mathcal{E}_{12}$ be fixed to $\Delta M_{12}^{\text {obs }}$, the observed value of the mass difference of particles 1 and 2. $\Delta M_{12}^{\text {obs }}$ is given in terms of a physical unit $l\left(\right.$ like $\left.\mathrm{MeV}^{-1}\right)$. Then, in terms of $x \equiv \frac{R}{l}$, the energy difference is 


$$
\Delta \mathcal{E}_{12}(g, x)=\left(\frac{f_{n_{1}}^{k_{1}}(g)-f_{n_{2}}^{k_{2}}(g)}{x}\right) \frac{1}{l}
$$

The equation

$$
\Delta \mathcal{E}_{12}(g, x)=\Delta M_{12}^{\text {obs }}
$$

implicitly determines the function $g(x)$, which in turn determines $\mu(x)=\mu(g(x))$ and $\mu_{s}(x)=\mu_{s}(g(x))$. We will refer to (4.4) and (4.8) as mass-matching conditions.

In practice, it is easier to determine $x$ as a function of $g$ : inverting (4.8), we obtain

$$
x(g)=\left(\frac{f_{n_{1}}^{k_{1}}(g)-f_{n_{2}}^{k_{2}}(g)}{\Delta E_{12}^{\mathrm{obs}}}\right) \frac{1}{l} .
$$

This enables us to express the mass difference of a particle $x$ and particle 2 in terms of the corresponding mass difference ratio $\mathcal{R}_{x}$ :

$$
\Delta \mathcal{E}_{x, 2}(g) \equiv \mathcal{E}_{n_{x}}^{k_{x}}(g)-\mathcal{E}_{n_{2}}^{k_{2}}(g)=\mathcal{R}_{x}(g) \Delta E_{12}^{\mathrm{obs}} .
$$

Finally, to determine the actual masses of our particles, we set the asymptotic value of $\mathcal{E}_{n_{2}}^{k_{2}}$ to the observed mass of that particle from experimental data. This amounts to fixing the value of the additive constant $C$, which is the only parameter left in the theory to adjust.

Thus, by choosing the observed masses of four particles as inputs, we have determined the four functions, namely, $\mu(x), \mu_{s}(x), g(x)$ and $C(x)$. This enables us to predict the absolute masses of the other particles unambiguously.

\section{RESULTS}

\section{A. Identification of the particles}

Once we have determined the variational Hamiltonian $\tilde{H}$ of (3.3), we proceed to compute its generalized eigenvalues and eigenvectors, and identify the quantum numbers associated with them. Parity is not a good symmetry of the Hamiltonian due to the cubic term in $H_{\mathrm{YM}}$ and the fermion curvature term $H_{c}$. We do note that the expectation value of the parity operator in any eigenstate does asymptote to \pm 1 in the large $g$ limit, but this value is sensitive to the variational ansatz. Thus we cannot assign parity unambiguously in the current level of approximation.

We can, however, make two important qualitative predictions from our model:

(1) The Hamiltonian is symmetric under charge conjugation, which takes $M_{i a} T_{a} \rightarrow-M_{i a} T_{a}^{*}$ and $Q \rightarrow i \gamma^{2} Q^{*}$. Therefore particles and their antiparticles have the same energy. Similarly, the expectation values of various other quantum numbers, namely, spin, isospin and hypercharge are well defined for all $g$, because the corresponding generators commute with $H$. This enables us to assign these quantum numbers to the states in our multiplets. As a first qualitative test of our model, we find that the quantum numbers assigned to the lowest flavor multiplets, in both the baryonic and mesonic sector, follow the same mass hierarchy, and exactly match with the corresponding particles observed in nature.

(2) Any variational eigenstate is a linear combination of states with different number of quarks and antiquarks. Nevertheless, the expectation value of the fermion number operator $N_{Q}$ in such an eigenstate is well defined. We find that in the large $g$ limit, the lowest flavor multiplet in the mesonic sector has $\left\langle N_{Q}\right\rangle=2.00042$ and in the baryonic sector has $\left\langle N_{Q}\right\rangle=3.00040$. We thus identify them with the lightest mesons and baryons respectively. We illustrate this for the pseudoscalar and vector meson octets in Fig. 1, and baryon octet and decuplet in Fig. 2.

\section{B. Mass-matching, predictions and error analysis}

Following the argument outlined in Sec. IV, we choose the observed masses of the pion, the $\rho$-meson, the $\Lambda$ baryon and the $\Delta$ baryon as inputs and solve (4.4) and (4.8). We take their experimental masses from the particle data booklet [31]: $m_{\pi}=138.04 \mathrm{MeV}, \quad m_{\rho}=775.16 \mathrm{MeV}$, $m_{\Lambda}=1115.16 \mathrm{MeV}$ and $m_{\Delta}=1232.00 \mathrm{MeV}$. Because we have taken the masses of the $u$ and $d$ quarks to be the same, isospin symmetry is exact, and particles in the same isospin multiplet have equal masses. Thus the experimental numbers quoted are the average value of the masses of all particles in the same isospin multiplet.

We first determine the functions $\mu(g)$ and $\mu_{s}(g)$ by fixing two mass-difference ratios to their experimental values:

$$
\begin{aligned}
& \mathcal{R}_{\rho \pi \Lambda}\left(\mu, \mu_{s}, g\right) \equiv \frac{f_{\Lambda}-f_{\pi}}{f_{\rho}-f_{\pi}}=1.534, \\
& \mathcal{R}_{\rho \pi \Delta}\left(\mu, \mu_{s}, g\right) \equiv \frac{f_{\Delta}-f_{\pi}}{f_{\rho}-f_{\pi}}=1.717 .
\end{aligned}
$$

Since (5.1) and (5.2) are nonlinear equations involving eigenvalues of very large matrices, it is not easy to obtain analytic solutions. However it is fairly easy to solve them numerically for a discrete set of values of $g$. As shown in Fig. 3, the functions $\mu(g)$ and $\mu_{s}(g)$ asymptote to definite values for large $g$.

These asymptotic values give the constituent quark masses in our model, in units of inverse $R$. The ratio of the constituent quark masses is independent of the unit chosen, and is found to be

$$
\frac{\mu_{s}}{\mu}=1.26
$$

With this choice of scaling functions $\mu(g)$ and $\mu_{s}(g)$, the other mass-difference ratios also asymptote to constant 


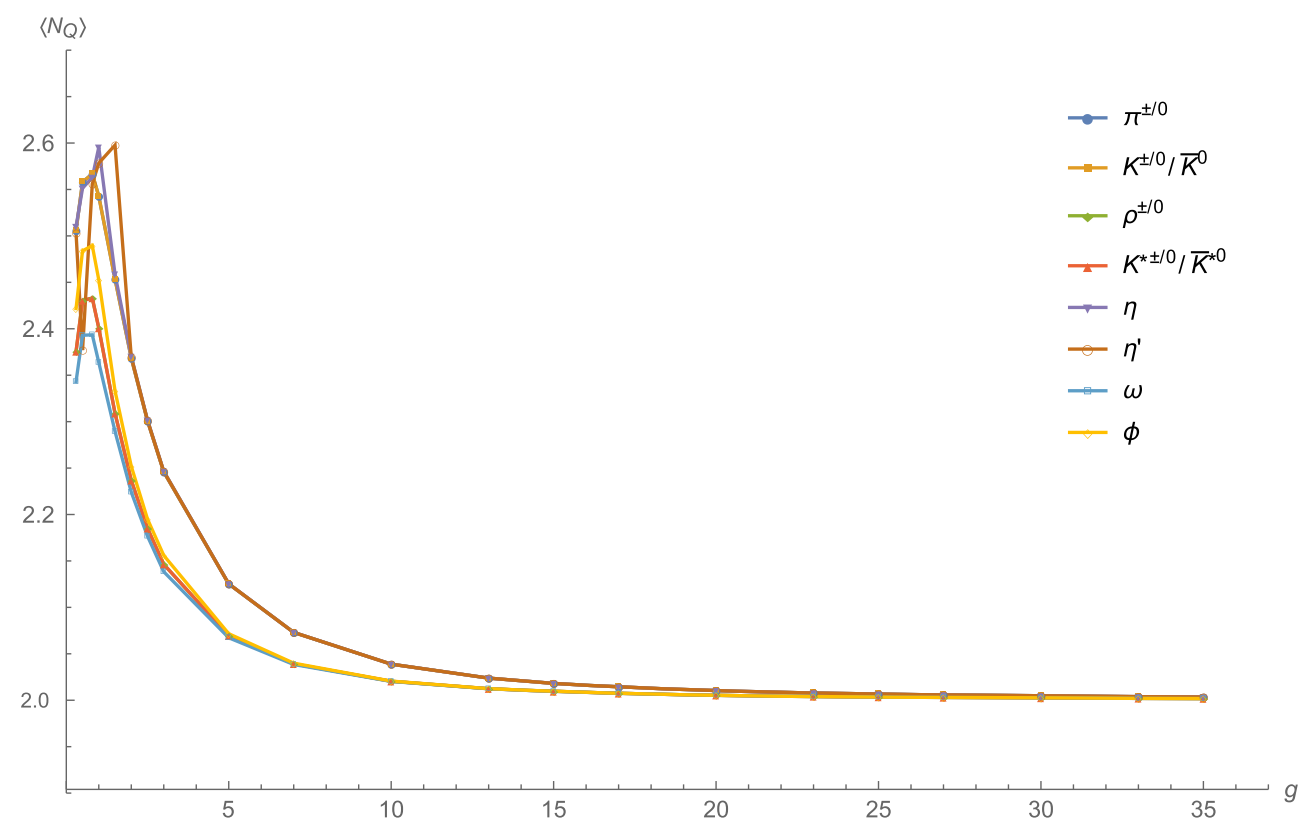

FIG. 1. A plot of $\left\langle N_{Q}\right\rangle$ vs $g$ for the mesons.

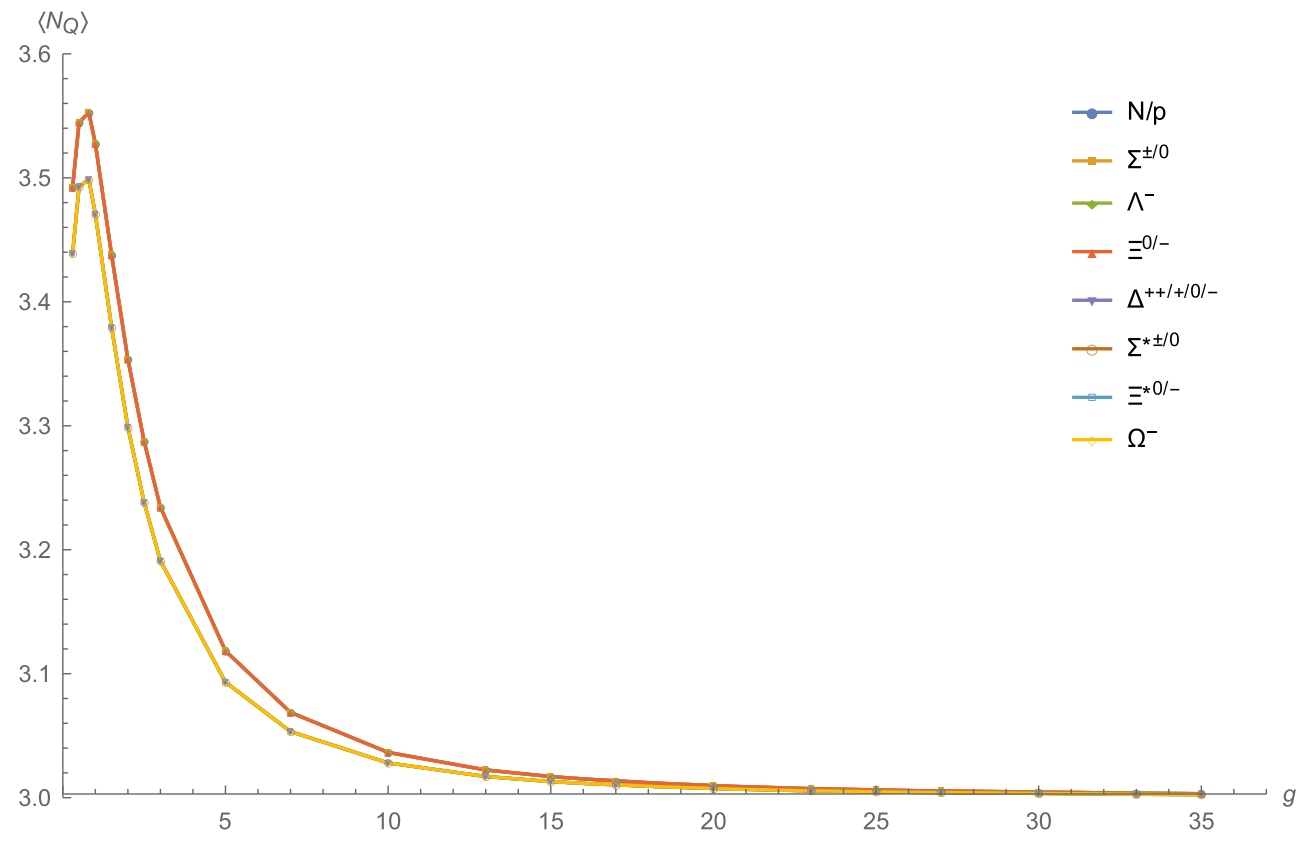

FIG. 2. A plot of $\left\langle N_{Q}\right\rangle$ vs $g$ for the baryons.

values. We demonstrate this graphically for a few of the particles in Fig. 4 below.

To determine the actual masses, we use $m_{\pi}=$ $138.04 \mathrm{MeV}$ and $m_{\rho}=775.16 \mathrm{MeV}$, and predict the masses of the other hadrons.

Before we report our predictions, we would like to say a few words about the choice of input masses and error analysis. Different sets of input particles lead to slightly different predictions for the masses of the other particles.
For each input set, we can compute the chi-squared $\left(\chi^{2}\right)$ value of our predictions, with $\chi^{2}$ defined as

$$
\chi^{2} \equiv \sum_{i} \frac{\left(M_{m m}^{i}-M_{\mathrm{obs}}^{i}\right)^{2}}{M_{\mathrm{obs}}^{i}}
$$

Here, $M_{m m}^{i}$ and $M_{\mathrm{obs}}^{i}$ are the predicted and observed masses of the $i$ th particle respectively. In Table I below, we provide 


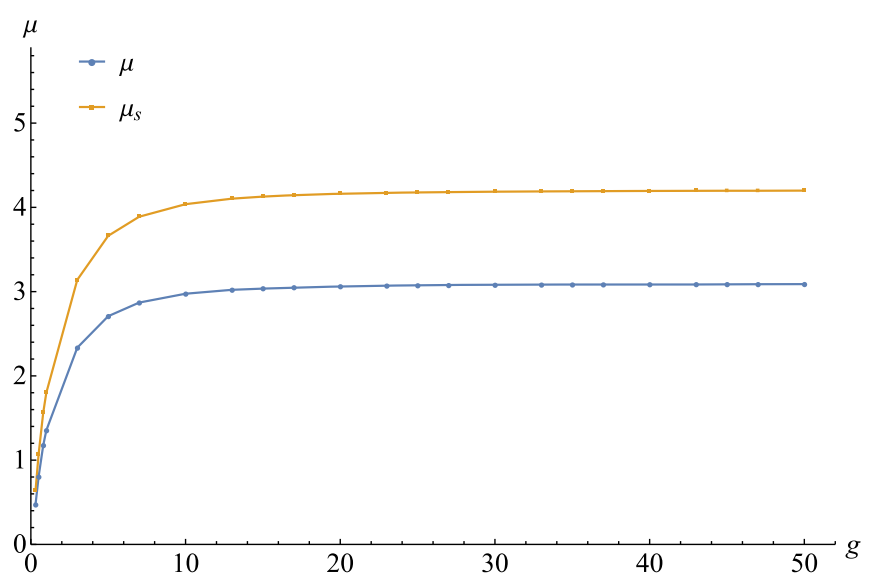

FIG. 3. A plot of $\mu$ and $\mu_{s}$ vs $g$.

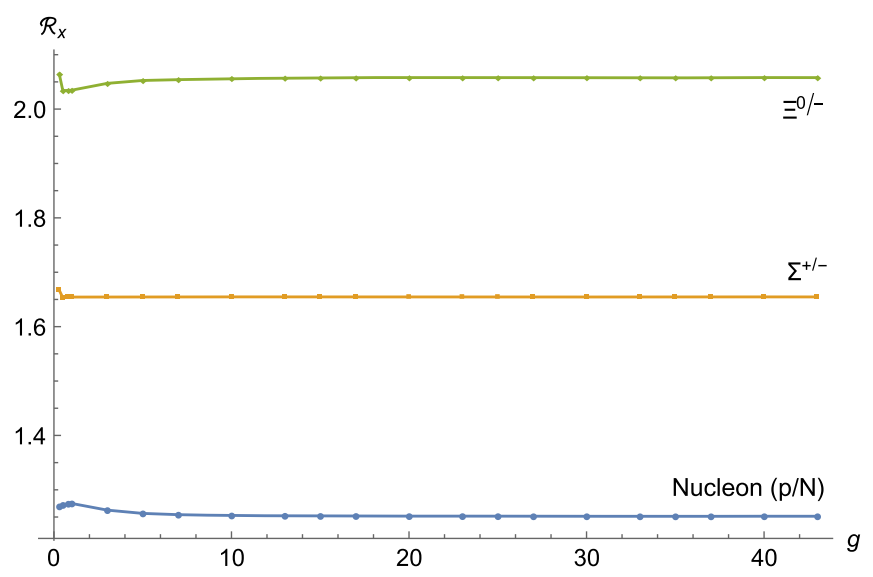

FIG. 4. Plot of $\mathcal{R}_{x}$ vs $g$ for some particles $x$. We show the asymptotic behavior of $\mathcal{R}_{x}$ for three of the baryons, namely, the nucleon, the $\Lambda^{0}$ and the $\Xi$ baryon.

a list of typical $\chi^{2}$ values for various choices of inputs. This justifies our choice of $\pi, \rho, \Lambda, \Delta$ as inputs because it minimizes $\chi^{2}$.

In the above discussion, we have set the values of our input masses to their exact quoted observed values. However, in principle, there are two important sources of mismatch of our model with nature. One source of mismatch is of course the truncation of our variational

TABLE I. A list of input sets giving lowest few values of $\chi^{2}$.

\begin{tabular}{lc}
\hline \hline$\pi, \rho, \Lambda, \Delta$ & 95.38 \\
\hline Inputs & $\chi^{2}(\mathrm{MeV})$ \\
$\pi, \rho, \Sigma^{*}, \Delta$ & 104.31 \\
$\pi, \rho, \Xi, \Delta$ & 107.29 \\
$\pi, \rho, \Xi^{*}, \Delta$ & 109.49 \\
$\pi, \rho, \Omega, \Delta$ & 126.09 \\
$\pi, \rho, \Lambda, \Xi$ & 132.17 \\
$\rho, K^{*}, \Lambda, \Delta$ & 191.13 \\
\hline \hline
\end{tabular}

ansatz at the level of four-boson and five-fermion states. The other source of mismatch lies in the fact that the matrix model is an approximation of the full QCD. These two sources of mismatch effectively induce error bars in the input masses, which in turn propagate and give error bars for our predictions.

Although quantitative error analysis of variational estimates is notoriously difficult, we can make a rough estimate of the input errors by varying our input set. We do this in the following way: we choose a larger set of input particles, namely, $\pi, \rho, \Lambda, \Delta, \Sigma^{*}$ and $\Xi$. Out of this set, we can make 15 independent choices of 4 inputs, each of which leads to a slightly different prediction for the mass of the rest of the particles in this set. This enables us to put error bars in the masses of our original choice of input particles, which we summarize in Table II.

With these error bars, the ratio of constituent quark masses is obtained as

$$
\frac{\mu_{s}}{\mu}=1.260 \pm 0.047 .
$$

\section{Results}

Our results are given in Tables III-V. In Table III, we report the asymptotic values of the mass difference ratios $\mathcal{R}_{x}$ for all the light mesons and baryons (except the ones used as inputs) from the matrix model, as well as their observed values. In Tables IV and V, we present the matrix

TABLE II. A list of input masses with error bars.

\begin{tabular}{lc}
\hline \hline Input particle & Mass with error bar $(\mathrm{MeV})$ \\
\hline$\pi^{ \pm / 0}$ & $138.04 \pm 37.88$ \\
$\rho^{ \pm / 0}$ & $775.16 \pm 51.53$ \\
$\Lambda^{-}$ & $1115.16 \pm 21.52$ \\
$\Delta^{++/+/ 0 /-}$ & $1232.00 \pm 28.18$ \\
\hline \hline
\end{tabular}

TABLE III. Mass-difference ratios with error bars for different mesons and baryons, and their observed values.

\begin{tabular}{lcc}
\hline \hline Particle $(\mathrm{x})$ & $\mathcal{R}_{x}=\frac{\mathcal{E}_{x}-\mathcal{E}_{\pi}}{\mathcal{E}_{\rho}-\mathcal{E}_{\pi}}$ & Observed value of $R_{x}=\frac{m_{x}-m_{\pi}}{m_{\rho}-m_{\pi}}$ \\
\hline$K^{ \pm} / K^{0} / \bar{K}^{0}$ & $0.303 \pm 0.035$ & 0.560 \\
$K^{* \pm} / K^{* 0} / \bar{K}^{* 0}$ & $1.303 \pm 0.035$ & 1.185 \\
$\eta$ & $0.001 \pm 0$ & 0.643 \\
$\eta^{\prime}$ & $0.606 \pm 0.070$ & 1.267 \\
$\omega$ & $1.001 \pm 0$ & 1.011 \\
$\phi$ & $1.606 \pm 0.070$ & 1.385 \\
$p / N$ & $1.318 \pm 0.184$ & 1.256 \\
$\Xi^{0 /-}$ & $1.924 \pm 0.200$ & 1.846 \\
$\Sigma^{0}$ & $1.621 \pm 0.188$ & 1.655 \\
$\Sigma^{*( \pm / 0)}$ & $2.087 \pm 0.188$ & 2.190 \\
$\Xi^{* 0 /-}$ & $2.390 \pm 0.199$ & 1.958 \\
$\Omega^{-}$ & $2.693 \pm 0.215$ & 2.408 \\
\hline \hline
\end{tabular}


TABLE IV. Comparison of the meson masses in the matrix model with data.

\begin{tabular}{lccccc}
\hline \hline Particle & Spin & Isospin & Matrix model mass $(\mathrm{MeV})$ & Observed mass $(\mathrm{MeV})$ & $\%$ Difference \\
\hline$K^{ \pm} / K^{0} / \bar{K}^{0}$ & 0 & $1 / 2$ & $331.09 \pm 48.06$ & 495.65 & $-33.2 \%$ \\
$\eta$ & 0 & 0 & $138.03 \pm 37.88$ & 547.86 & $-74.8 \%$ \\
$\eta^{\prime}$ & 0 & 0 & $524.14 \pm 70.23$ & 957.78 & $-45.3 \%$ \\
$K^{* \pm} / K^{* 0} / \bar{K}^{* 0}$ & 1 & $1 / 2$ & $968.21 \pm 94.22$ & 893.65 & $+8.3 \%$ \\
$\omega$ & 1 & 0 & $775.19 \pm 74.33$ & 782.65 & $-0.9 \%$ \\
$\phi$ & 1 & 0 & $1161.24 \pm 118.23$ & 1019.46 & $+13.9 \%$ \\
\hline \hline
\end{tabular}

TABLE V. Comparison of the baryon masses in the matrix model with data.

\begin{tabular}{lccccc}
\hline \hline Particle & Spin & Isospin & Matrix model mass $(\mathrm{MeV})$ & Observed mass $(\mathrm{MeV})$ & $\%$ Difference \\
\hline$p / N$ & $1 / 2$ & $1 / 2$ & $977.74 \pm 149.24$ & 938.91 & $+4.1 \%$ \\
$\Xi^{0 /-}$ & $1 / 2$ & $1 / 2$ & $1363.85 \pm 180.69$ & 1314.86 & $+3.7 \%$ \\
$\Sigma^{( \pm / 0)}$ & $1 / 2$ & 0 & $1170.79 \pm 163.05$ & 1193.15 & $-1.9 \%$ \\
$\Sigma^{*( \pm / 0)}$ & $3 / 2$ & 1 & $1467.84 \pm 183.46$ & 1384.6 & $+6.0 \%$ \\
$\Xi^{* 0 /-}$ & $3 / 2$ & $1 / 2$ & $1660.89 \pm 202.18$ & 1533.4 & $+8.3 \%$ \\
$\Omega^{-}$ & $3 / 2$ & 0 & $1853.94 \pm 223.25$ & 1672.45 & $+10.8 \%$ \\
\hline \hline
\end{tabular}

model predictions for the light meson and baryon masses from our model and compare these with the observed values taken from the particle data booklet [31]. We also give the percentage error by which our results (central values) differ from the observed values. Figure 5 gives a summary of our results.

\section{DISCUSSION}

We observe that the matrix model predicts light hadron masses with fair accuracy, with most masses lying within $20 \%$ of their observed values. The predictions for baryon masses are especially good, with surprisingly small errors.

We do not make a correct prediction for the masses of the $\eta$ and $\eta^{\prime}$ mesons: their values are underestimated by about $300 \mathrm{MeV}$. The root of this issue lies in the fact that the matrix model predicts a different quark content for the $\eta$

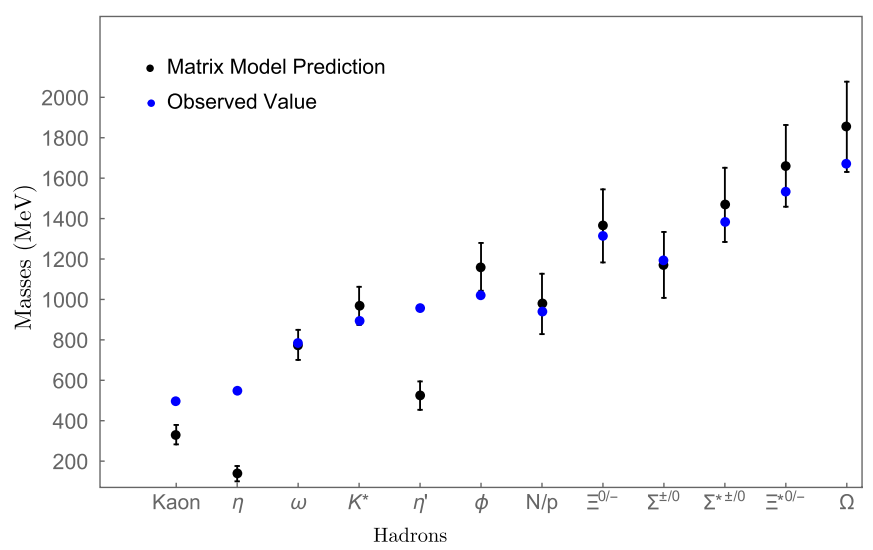

FIG. 5. Matrix model estimates of the light hadron masses compared with observed values. and $\eta^{\prime}$ mesons from the Standard Model. To elaborate, the quark model predicts the particles

$$
\eta_{8}=\frac{1}{\sqrt{6}}(u \bar{u}+d \bar{d}-2 s \bar{s})
$$

and

$$
\eta_{1}=\frac{1}{\sqrt{3}}(u \bar{u}+d \bar{d}+s \bar{s})
$$

The $\eta$ and $\eta^{\prime}$ mesons are expressed as the linear combination

$$
\left(\begin{array}{c}
\eta \\
\eta^{\prime}
\end{array}\right)=\left(\begin{array}{cc}
\cos \theta_{P} & -\sin \theta_{P} \\
\sin \theta_{P} & \cos \theta_{P}
\end{array}\right)\left(\begin{array}{l}
\eta_{8} \\
\eta_{1}
\end{array}\right)
$$

where $\theta_{P} \sim 13.3^{\circ}$ is the mixing angle [32]. The mass eigenstates corresponding to $\eta, \eta^{\prime}$ in our matrix model, however, have a different quark content, which is roughly

$$
\eta_{m m}=\frac{1}{\sqrt{2}}(u \bar{u}+d \bar{d}), \quad \eta_{m m}^{\prime}=s \bar{s} .
$$

This discrepancy occurs because the matrix model seems to be insensitive to the effect of the $U(1)_{A}$ axial anomaly [33-35] at the current level of approximation. As stated in $[36,37]$, if the axial anomaly-induced effective gluon mass term is too small, the eigenstates of the mass matrix correspond to hypothetical particles $\eta_{q}$ and $\eta_{s}$, which have the same quark content as $\eta_{m m}$ and $\eta_{m m}^{\prime}$ respectively in (6.4), their masses being 
TABLE VI. Comparison of the $\eta$ and $\eta^{\prime}$ masses in the matrix model with the $\eta_{q}$ and $\eta_{s}$ masses.

\begin{tabular}{cccccc}
\hline \hline \multicolumn{6}{c}{ Matrix model } \\
Particle Spin Isospin & $\begin{array}{c}\text { Observed } \\
\text { mass }(\mathrm{MeV})\end{array}$ & mass (MeV) & Difference \\
\hline$\eta_{q}$ & 0 & 0 & $138.03 \pm 37.88$ & 138.04 & $0.0 \%$ \\
$\eta_{s}$ & 0 & 0 & $524.14 \pm 70.23$ & 687.23 & $-23.7 \%$ \\
\hline \hline & $M_{q}=m_{\pi}$, \\
& $M_{s}=\sqrt{2 m_{K}^{2}-m_{\pi}^{2}}$,
\end{tabular}

where $m_{\pi}$ and $m_{K}$ are the masses of the pion and kaon respectively. Therefore, the masses of the $\eta$ and $\eta^{\prime}$ meson predicted from the matrix model should really be compared with the masses of the hypothetical $\eta_{q}$ and $\eta_{s}$ mesons from (6.6). This comparison is given in Table VI below.

This is an issue that requires further investigation and for this reason, we have excluded the $\eta$ and $\eta^{\prime}$ meson in the calculation of $\chi^{2}$ in Table I.

At this level of approximation, we have considered variational states with up to only 4 bosonic oscillators and 4 fermions. Our use of resources, both computational and human, has been modest.

Our estimates are expected to improve if we increase the size of our variational trial set by including states with up to 6 bosonic oscillators and 7 fermions. This larger trial set has several thousand states and involves computations with very large matrices. This requires more efficient numerical codes to be developed for the matrix element computation as well as enumeration of states, and is the focus of our future research.
The next lowest flavor multiplet in the mesonic sector has $\left\langle N_{Q}\right\rangle=3.9999$, and in the baryonic sector has $\left\langle N_{Q}\right\rangle=4.9999$, as seen from Fig. 6. These are candidates for light exotic hadrons, i.e., hadrons with more than three quarks [38]. These are tetraquark and pentaquark states, with quark content $q q \bar{q} \bar{q}$ and $q q q q \bar{q}$ respectively. Light tetraquarks were first discussed by Jaffe [39] in the context of the bag model $[40,41]$, where he suggested the existence of a tetraquark nonet with mass below $1 \mathrm{GeV}$, and proposed this as a model for the light scalar mesons. The matrix model prediction for the masses of these particles are about $2.5 \mathrm{GeV}$. This estimate is expected to improve considerably when we include more states in the trial set.

\section{APPENDIX: VARIATIONAL STATES FOR THE LIGHT HADRONS}

\section{Fermionic states}

We denote the fermion vacuum by $\left|0_{F}\right\rangle$. Fermionic states can be created by acting on $\left|0_{F}\right\rangle$ successively by the fermion or the antifermion creation operator $b^{\dagger}$ and $d^{\dagger}$ (with the indices suppressed for convenience). A fermionic state can be assigned the quantum numbers $(f, s, c)$, denoting its representation under flavor $S U(3)$, spin and representation under color $S U(3)$ respectively. A quark state, $b^{\dagger}\left|0_{F}\right\rangle$, is denoted by the quantum numbers $\left(\mathbf{3}, \frac{1}{2}, \mathbf{3}\right)$ and an antiquark, $d^{\dagger}\left|0_{F}\right\rangle$ by $\left(\overline{\mathbf{3}}, \frac{1}{\mathbf{2}}, \overline{\mathbf{3}}\right)$.

A "mesonic" state is created by acting on $\left|0_{F}\right\rangle$ by a pair of quark and antiquark creation operator. Taking a tensor product of the representations gives us all possible ways such a state can transform;

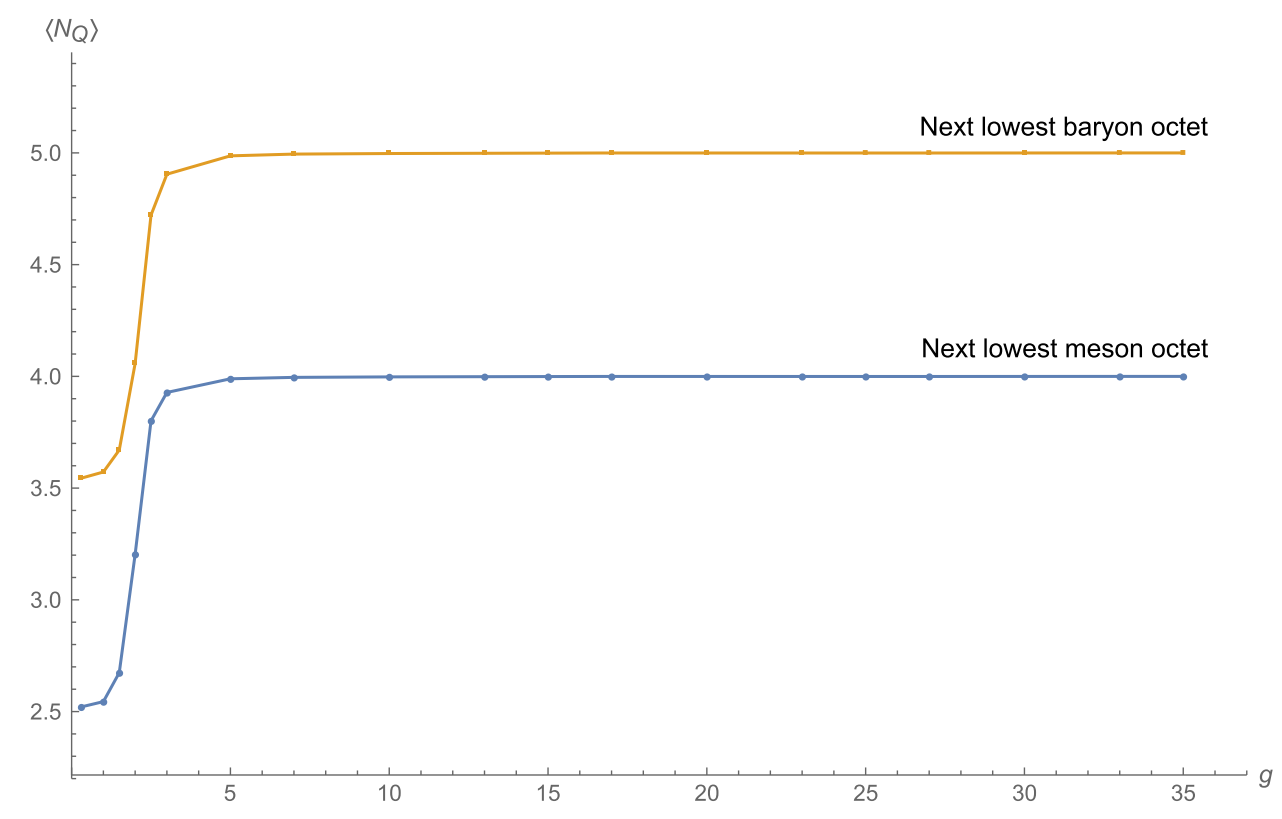

FIG. 6. A plot of $\left\langle N_{Q}\right\rangle$ vs $g$ for the next lowest meson and baryon octet. 


$$
\left(3, \frac{1}{2}, 3\right) \otimes\left(\overline{3}, \frac{1}{2}, \overline{3}\right)=(8 \oplus 1,1 \oplus 0,8 \oplus 1) .
$$

This gives the eight possible mesonic states in the ansatz, transforming as $(\mathbf{1}, \mathbf{0}, \mathbf{1}),(\mathbf{1}, \mathbf{1}, \mathbf{1}),(\mathbf{1}, \mathbf{0}, \mathbf{8}),(\mathbf{1}, \mathbf{1}, \mathbf{8})$, $(\mathbf{8}, \mathbf{0}, \mathbf{1}),(\mathbf{8}, \mathbf{1}, \mathbf{1}),(\mathbf{8}, \mathbf{0}, \mathbf{8})$ and $(\mathbf{8}, \mathbf{1}, \mathbf{8})$ respectively.

The interaction term of the Hamiltonian connects a mesonic state to a state with a pair of mesons. Such states can be created by applying on the vacuum two successive meson creation operators. A tensor product decomposition of two mesonic representations gives

$$
\begin{aligned}
{\left[\left(\mathbf{3}, \frac{\mathbf{1}}{\mathbf{2}}, \mathbf{3}\right) \otimes\left(\overline{\mathbf{3}}, \frac{\mathbf{1}}{\mathbf{2}}, \overline{\mathbf{3}}\right)\right]^{2} } \\
=(\mathbf{8} \oplus \mathbf{1}, \mathbf{1} \oplus \mathbf{0}, \mathbf{8} \oplus \mathbf{1}) \otimes(\mathbf{8} \oplus \mathbf{1}, \mathbf{1} \oplus \mathbf{0}, \mathbf{8} \oplus \mathbf{1}) \\
=(\mathbf{2 7} \oplus \mathbf{1 0} \oplus \overline{\mathbf{1 0}} \oplus 4 \mathbf{8} \oplus 2 \mathbf{1}, \mathbf{2} \oplus 3 \mathbf{1} \oplus 2 \mathbf{0}, 27 \\
\quad \oplus \mathbf{1 0} \oplus \overline{\mathbf{1 0}} \oplus 4 \mathbf{8} \oplus 2 \mathbf{1}) .
\end{aligned}
$$

Since the interaction term is a flavor singlet, only those states contribute to a given meson mass whose flavor quantum number is the same as the corresponding meson state. So, we are only interested in the states with $f=1$ or 8 .

The above counting actually gives more states in a particular representation than are actually in the ansatz. For example, looking at (A2), there seem to be 16 possible states transforming as $(\mathbf{8 , 0}, \mathbf{1})$. However, only 4 of them are linearly independent.

A "baryonic" state is created by acting on $\left|0_{F}\right\rangle$ by three quark creation operators. Taking a tensor product of three quark representations, we find

$$
\begin{aligned}
\left(3, \frac{1}{2}, 3\right) \otimes\left(3, \frac{1}{2}, 3\right) \otimes\left(3, \frac{1}{2}, 3\right) \\
=\left(10 \oplus 28 \oplus 1, \frac{3}{2} \oplus 2 \frac{1}{2}, 10 \oplus 28 \oplus 1\right) .
\end{aligned}
$$

These states are also organized according to their flavor quantum number. In particular, we organize the states into a baryon decuplet and a baryon octet.

The interaction term $H_{\text {int }}$ in (2.21) connects a baryonic state to a state created by applying on $\left|0_{F}\right\rangle$ a baryon and a meson creation operator successively. Again, a naive counting by taking the tensor product of representations gives many more states than are linearly independent.
A list of all the linearly independent fermionic states used to create the variational ansatz for light hadrons is given below. Fermionic states are organized according to their quantum numbers $(f, s, c)$. According to the quark content, the states are referred to as mesonic, di-mesonic, baryonic and meson-baryon states. Generators of rotation are denoted by $\tau_{i}=\frac{1}{2} \sigma_{i}$ and generators of color by $T_{a}=\frac{1}{2} \lambda_{a}$. Also, generators of the flavor symmetry are denoted by $\mathcal{T}_{F}=\frac{1}{2} \lambda_{F}$.

For notational convenience, spin-2 states are written with two free adjoint indices $i, j$. A spin-2 state can be constructed from a state $\left|\psi_{i j}\right\rangle$ with two free adjoint spin indices as

$$
\left|\psi_{i j}^{(2)}\right\rangle=\frac{1}{2}\left(\left|\psi_{i j}\right\rangle+\left|\psi_{j i}\right\rangle\right)-\frac{1}{3} \delta_{i j}\left|\psi_{k k}\right\rangle .
$$

Similarly, a state transforming in the representation $\mathbf{2 7 , 1 0}$ or $\overline{\mathbf{1 0}}$ under color is also written with two free adjoint tensors. The states with definite color can be constructed as follows:

$\left|\psi_{a b}^{(27)}\right\rangle=\frac{1}{2}\left(\left|\psi_{a b}\right\rangle+\left|\psi_{b a}\right\rangle\right)-\frac{3}{5}\left(d_{c d e}\left|\psi_{d e}\right\rangle\right) d_{a b c}-\frac{1}{8} \delta_{a b}\left|\psi_{c c}\right\rangle$,

$\left|\psi_{a b}^{(20)}\right\rangle=\frac{1}{2}\left(\left|\psi_{a b}\right\rangle-\left|\psi_{b a}\right\rangle\right)-\frac{1}{3}\left(f_{c d e}\left|\psi_{d e}\right\rangle\right) f_{a b c}$.

Note that since the $\mathbf{1 0}$ and $\overline{\mathbf{1 0}}$ representations are conjugate to each other and orthogonal, they can be combined into a $\mathbf{2 0} \equiv \mathbf{1 0} \oplus \overline{\mathbf{1 0}}$.

Meson and di-meson states are organized under flavor $S U(3)$ as an octet or a singlet.
(1) $(\mathbf{1}, \mathbf{0}, \mathbf{1}): b_{f \alpha A}^{\dagger}(\epsilon c)_{f \alpha A}^{\dagger}\left|0_{F}\right\rangle$
(2) $(\mathbf{1}, \mathbf{1}, \mathbf{1}): b_{f \alpha A}^{\dagger}\left(\tau_{i}\right)_{\alpha \beta}(\epsilon c)_{f \beta A}^{\dagger}\left|0_{F}\right\rangle$
(3) $(\mathbf{1 , 0 , 8}): b_{f \alpha A}^{\dagger}\left(T_{a}\right)_{A B}(\epsilon c)_{f \alpha B}^{\dagger}\left|0_{F}\right\rangle$
(4) $(\mathbf{1}, \mathbf{1}, \mathbf{8}): b_{f \alpha A}^{\dagger}\left(\tau_{i}\right)_{\alpha \beta}\left(T_{a}\right)_{A B}(\epsilon c)_{f \beta B}^{\dagger}\left|0_{F}\right\rangle$
(5) $(\mathbf{8 , 0}, \mathbf{1}):\left(\mathcal{T}_{F}\right)_{f g} b_{f \alpha A}^{\dagger}(\epsilon c)_{g \alpha A}^{\dagger}\left|0_{F}\right\rangle$
(6) $(\mathbf{8}, \mathbf{1}, \mathbf{1}):\left(\mathcal{T}_{F}\right)_{f g} b_{f \alpha A}^{\dagger}\left(\tau_{i}\right)_{\alpha \beta}(\epsilon c)_{g \beta A}^{\dagger}\left|0_{F}\right\rangle$
(7) $(\mathbf{8 , 0}, \mathbf{8}):\left(\mathcal{T}_{F}\right)_{f g} b_{f \alpha A}^{\dagger}\left(T_{a}\right)_{A B}(\epsilon c)_{g \alpha B}^{\dagger}\left|0_{F}\right\rangle$
(8) $(\mathbf{8}, \mathbf{1}, \mathbf{8}):\left(\mathcal{T}_{F}\right)_{f g} b_{f \alpha A}^{\dagger}\left(\tau_{i}\right)_{\alpha \beta}\left(T_{a}\right)_{A B}(\epsilon c)_{g \beta B}^{\dagger}\left|0_{F}\right\rangle$

\section{b. Di-meson states}

(1) $(\mathbf{1}, \mathbf{0}, \mathbf{1})$ :
(a) $b_{f_{1} \alpha_{1} A_{1}}^{\dagger}(\epsilon c)_{f_{1} \alpha_{1} A_{1}}^{\dagger} b_{f_{2} \alpha_{2} A_{2}}^{\dagger}(\epsilon c)_{f_{2} \alpha_{2} A_{2}}^{\dagger}\left|0_{F}\right\rangle$
(b) $b_{f_{1} \alpha_{1} A_{1}}^{\dagger}\left(\tau_{i}\right)_{\alpha_{1} \beta_{1}}(\epsilon c)_{f_{1} \beta_{1} A_{1}}^{\dagger} b_{f_{2} \alpha_{2} A_{2}}^{\dagger}\left(\tau_{i}\right)_{\alpha_{2} \beta_{2}}(\epsilon c)_{f_{2} \beta_{2} A_{2}}^{\dagger}\left|0_{F}\right\rangle$
(c) $b_{f_{1} \alpha_{1} A_{1}}^{\dagger}\left(T_{a}\right)_{A_{1} B_{1}}(\epsilon c)_{f_{1} \alpha_{1} B_{1}}^{\dagger} b_{f_{2} \alpha_{2} A_{2}}^{\dagger}\left(T_{a}\right)_{A_{2} B_{2}}(\epsilon c)_{f_{2} \alpha_{2} A_{2}}^{\dagger}\left|0_{F}\right\rangle$
(d) $b_{f_{1} \alpha_{1} A_{1}}^{\dagger}\left(\tau_{i}\right)_{\alpha_{1} \beta_{1}}\left(T_{a}\right)_{A_{1} B_{1}}(\epsilon c)_{f_{1} \beta_{1} B_{1}}^{\dagger} b_{f_{2} \alpha_{2} A_{2}}^{\dagger}\left(\tau_{i}\right)_{\alpha_{2} \beta_{2}}\left(T_{a}\right)_{A_{2} B_{2}}(\epsilon c)_{f_{2} \beta_{2} B_{2}}^{\dagger}\left|0_{F}\right\rangle$ 
(2) $(\mathbf{1}, \mathbf{1}, \mathbf{1})$ :

(a) $b_{f_{1} \alpha_{1} A_{1}}^{\dagger}\left(\tau_{i}\right)_{\alpha_{1} \beta_{1}}(\epsilon c)_{f_{1} \beta_{1} A_{1}}^{\dagger} b_{f_{2} \alpha_{2} A_{2}}^{\dagger}(\epsilon c)_{f_{2} \alpha_{2} A_{2}}^{\dagger}\left|0_{F}\right\rangle$

(b) $b_{f_{1} \alpha_{1} A_{1}}^{\dagger}\left(\tau_{i}\right)_{\alpha_{1} \beta_{1}}\left(T_{a}\right)_{A_{1} B_{1}}(\epsilon c)_{f_{1} \beta_{1} B_{1}}^{\dagger} b_{f_{2} \alpha_{2} A_{2}}^{\dagger}\left(T_{a}\right)_{A_{2} B_{2}}(\epsilon c)_{f_{2} \alpha_{2} A_{2}}^{\dagger}\left|0_{F}\right\rangle$

(3) $(\mathbf{1}, \mathbf{2}, \mathbf{1})$ :

(a) $b_{f_{1} \alpha_{1} A_{1}}^{\dagger}\left(\tau_{i}\right)_{\alpha_{1} \beta_{1}}(\epsilon c)_{f_{1} \beta_{1} A_{1}}^{\dagger} b_{f_{2} \alpha_{2} A_{2}}^{\dagger}\left(\tau_{j}\right)_{\alpha_{2} \beta_{2}}(\epsilon c)_{f_{2} \beta_{2} A_{2}}^{\dagger}\left|0_{F}\right\rangle$

(b) $b_{f_{1} \alpha_{1} A_{1}}^{\dagger}\left(\tau_{i}\right)_{\alpha_{1} \beta_{1}}\left(T_{a}\right)_{A_{1} B_{1}}(\epsilon c)_{f_{1} \beta_{1} B_{1}}^{\dagger} b_{f_{2} \alpha_{2} A_{2}}^{\dagger}\left(\tau_{j}\right)_{\alpha_{2} \beta_{2}}\left(T_{a}\right)_{A_{2} B_{2}}(\epsilon c)_{f_{2} \beta_{2} A_{2}}^{\dagger}\left|0_{F}\right\rangle$

(4) $(\mathbf{1}, \mathbf{0}, \mathbf{8})$ :

(a) $b_{f_{1} \alpha_{1} A_{1}}^{\dagger}(\epsilon c)_{f_{1} \alpha_{1} A_{1}}^{\dagger} b_{f_{2} \alpha_{2} A_{2}}^{\dagger}\left(T_{a}\right)_{A_{2} B_{2}}(\epsilon c)_{f_{2} \alpha_{2} B_{2}}^{\dagger}\left|0_{F}\right\rangle$

(b) $d_{a b c} b_{f_{1} \alpha_{1} A_{1}}^{\dagger}\left(T_{b}\right)_{A_{1} B_{1}}(\epsilon c)_{f_{1} \alpha_{1} B_{1}}^{\dagger} b_{f_{2} \alpha_{2} A_{2}}^{\dagger}\left(T_{c}\right)_{A_{2} B_{2}}(\epsilon c)_{f_{2} \alpha_{2} B_{2}}^{\dagger}\left|0_{F}\right\rangle$

(c) $b_{f_{1} \alpha_{1} A_{1}}^{\dagger}\left(\tau_{i}\right)_{\alpha_{1} \beta_{1}}(\epsilon c)_{f_{1} \beta_{1} A_{1}}^{\dagger} b_{f_{2} \alpha_{2} A_{2}}^{\dagger}\left(\tau_{i}\right)_{\alpha_{2} \beta_{2}}\left(T_{a}\right)_{A_{2} B_{2}}(\epsilon c)_{f_{2} \beta_{2} B_{2}}^{\dagger}\left|0_{F}\right\rangle$

(d) $d_{a b c} b_{f_{1} \alpha_{1} A_{1}}^{\dagger}\left(\tau_{i}\right)_{\alpha_{1} \beta_{1}}\left(T_{b}\right)_{A_{1} B_{1}}(\epsilon c)_{f_{1} \beta_{1} B_{1}}^{\dagger} b_{f_{2} \alpha_{2} A_{2}}^{\dagger}\left(\tau_{i}\right)_{\alpha_{2} \beta_{2}}\left(T_{c}\right)_{A_{2} B_{2}}(\epsilon c)_{f_{2} \beta_{2} B_{2}}^{\dagger}\left|0_{F}\right\rangle$

(5) $(\mathbf{1}, \mathbf{1}, \mathbf{8})$ :

(a) $b_{f_{1} \alpha_{1} A_{1}}^{\dagger}\left(\tau_{i}\right)_{\alpha_{1} \beta_{1}}\left(T_{a}\right)_{A_{1} B_{1}}(\epsilon c)_{f_{1} \beta_{1} B_{1}}^{\dagger} b_{f_{2} \alpha_{2} A_{2}}^{\dagger}(\epsilon c)_{f_{2} \alpha_{2} A_{2}}^{\dagger}\left|0_{F}\right\rangle$

(b) $b_{f_{1} \alpha_{1} A_{1}}^{\dagger}\left(\tau_{i}\right)_{\alpha_{1} \beta_{1}}(\epsilon c)_{f_{1} \beta_{1} A_{1}}^{\dagger} b_{f_{2} \alpha_{2} A_{2}}^{\dagger}\left(T_{a}\right)_{A_{2} B_{2}}(\epsilon c)_{f_{2} \alpha_{2} B_{2}}^{\dagger}\left|0_{F}\right\rangle$

(c) $f_{a b c} b_{f_{1} \alpha_{1} A_{1}}^{\dagger}\left(\tau_{i}\right)_{\alpha_{1} \beta_{1}}\left(T_{b}\right)_{A_{1} B_{1}}(\epsilon c)_{f_{1} \beta_{1} B_{1}}^{\dagger} b_{f_{2} \alpha_{2} A_{2}}^{\dagger}\left(T_{c}\right)_{A_{2} B_{2}}(\epsilon c)_{f_{2} \alpha_{2} A_{2}}^{\dagger}\left|0_{F}\right\rangle$

(d) $d_{a b c} b_{f_{1} \alpha_{1} A_{1}}^{\dagger}\left(\tau_{i}\right)_{\alpha_{1} \beta_{1}}\left(T_{b}\right)_{A_{1} B_{1}}(\epsilon c)_{f_{1} \beta_{1} B_{1}}^{\dagger} b_{f_{2} \alpha_{2} A_{2}}^{\dagger}\left(T_{c}\right)_{A_{2} B_{2}}(\epsilon c)_{f_{2} \alpha_{2} A_{2}}^{\dagger}\left|0_{F}\right\rangle$

(e) $\epsilon_{i j k} b_{f_{1} \alpha_{1} A_{1}}^{\dagger}\left(\tau_{j}\right)_{\alpha_{1} \beta_{1}}\left(T_{a}\right)_{A_{1} B_{1}}(\epsilon c)_{f_{1} \beta_{1} B_{1}}^{\dagger} b_{f_{2} \alpha_{2} A_{2}}^{\dagger}\left(\tau_{k}\right)_{\alpha_{2} \beta_{2}}(\epsilon c)_{f_{2} \beta_{2} A_{2}}^{\dagger}\left|0_{F}\right\rangle$

(f) $\epsilon_{i j k} f_{a b c} b_{f_{1} \alpha_{1} A_{1}}^{\dagger}\left(\tau_{j}\right)_{\alpha_{1} \beta_{1}}\left(T_{b}\right)_{A_{1} B_{1}}(\epsilon c)_{f_{1} \beta_{1} B_{1}}^{\dagger} b_{f_{2} \alpha_{2} A_{2}}^{\dagger}\left(\tau_{k}\right)_{\alpha_{2} \beta_{2}}\left(T_{c}\right)_{A_{2} B_{2}}(\epsilon c)_{f_{2} \beta_{2} B_{2}}^{\dagger}\left|0_{F}\right\rangle$

(6) $(\mathbf{1}, \mathbf{2}, \mathbf{8})$ :

(a) $b_{f_{1} \alpha_{1} A_{1}}^{\dagger}\left(\tau_{i}\right)_{\alpha_{1} \beta_{1}}\left(T_{a}\right)_{A_{1} B_{1}}(\epsilon c)_{f_{1} \beta_{1} B_{1}}^{\dagger} b_{f_{2} \alpha_{2} A_{2}}^{\dagger}\left(\tau_{j}\right)_{\alpha_{2} \beta_{2}}(\epsilon c)_{f_{2} \beta_{2} A_{2}}^{\dagger}\left|0_{F}\right\rangle$

(b) $d_{a b c} b_{f_{1} \alpha_{1} A_{1}}^{\dagger}\left(\tau_{i}\right)_{\alpha_{1} \beta_{1}}\left(T_{b}\right)_{A_{1} B_{1}}(\epsilon c)_{f_{1} \beta_{1} B_{1}}^{\dagger} b_{f_{2} \alpha_{2} A_{2}}^{\dagger}\left(\tau_{j}\right)_{\alpha_{2} \beta_{2}}\left(T_{c}\right)_{A_{2} B_{2}}(\epsilon c)_{f_{2} \beta_{2} A_{2}}^{\dagger}\left|0_{F}\right\rangle$

(7) $(\mathbf{1}, \mathbf{0}, 27 \oplus \mathbf{2 0})$ :

(a) $b_{f_{1} \alpha_{1} A_{1}}^{\dagger}\left(T_{a}\right)_{A_{1} B_{1}}(\epsilon c)_{f_{1} \alpha_{1} B_{1}}^{\dagger} b_{f_{2} \alpha_{2} A_{2}}^{\dagger}\left(T_{b}\right)_{A_{2} B_{2}}(\epsilon c)_{f_{2} \alpha_{2} B_{2}}^{\dagger}\left|0_{F}\right\rangle$

(b) $b_{f_{1} \alpha_{1} A_{1}}^{\dagger}\left(\tau_{i}\right)_{\alpha_{1} \beta_{1}}\left(T_{a}\right)_{A_{1} B_{1}}(\epsilon c)_{f_{1} \beta_{1} A_{1}}^{\dagger} b_{f_{2} \alpha_{2} A_{2}}^{\dagger}\left(\tau_{i}\right)_{\alpha_{2} \beta_{2}}\left(T_{b}\right)_{A_{1} B_{2}}(\epsilon c)_{f_{2} \beta_{2} B_{2}}^{\dagger}\left|0_{F}\right\rangle$

(8) $(\mathbf{1}, \mathbf{1}, 27 \oplus \mathbf{2 0})$ :

(a) $b_{f_{1} \alpha_{1} A_{1}}^{\dagger}\left(\tau_{i}\right)_{\alpha_{1} \beta_{1}}\left(T_{a}\right)_{A_{1} B_{1}}(\epsilon c)_{f_{1} \beta_{1} B_{1}}^{\dagger} b_{f_{2} \alpha_{2} A_{2}}^{\dagger}\left(T_{b}\right)_{A_{2} B_{2}}(\epsilon c)_{f_{2} \alpha_{2} B_{2}}^{\dagger}\left|0_{F}\right\rangle$

(b) $\epsilon_{i j k} b_{f_{1} \alpha_{1} A_{1}}^{\dagger}\left(\tau_{j}\right)_{\alpha_{1} \beta_{1}}\left(T_{a}\right)_{A_{1} B_{1}}(\epsilon c)_{f_{1} \beta_{1} A_{1}}^{\dagger} b_{f_{2} \alpha_{2} A_{2}}^{\dagger}\left(\tau_{k}\right)_{\alpha_{2} \beta_{2}}\left(T_{b}\right)_{A_{1} B_{2}}(\epsilon c)_{f_{2} \beta_{2} B_{2}}^{\dagger}\left|0_{F}\right\rangle$

(9) $(\mathbf{1}, \mathbf{2}, \mathbf{2 7} \oplus \mathbf{2 0})$ :

(a) $b_{f_{1} \alpha_{1} A_{1}}^{\dagger}\left(\tau_{i}\right)_{\alpha_{1} \beta_{1}}\left(T_{a}\right)_{A_{1} B_{1}}(\epsilon c)_{f_{1} \beta_{1} A_{1}}^{\dagger} b_{f_{2} \alpha_{2} A_{2}}^{\dagger}\left(\tau_{j}\right)_{\alpha_{2} \beta_{2}}\left(T_{b}\right)_{A_{1} B_{2}}(\epsilon c)_{f_{2} \beta_{2} B_{2}}^{\dagger}\left|0_{F}\right\rangle$

(10) $(\mathbf{8 , 0}, \mathbf{1})$ :

(a) $b_{f_{1} \alpha_{1} A_{1}}^{\dagger}\left(\mathcal{T}_{F}\right)_{f_{1} g_{1}}(\epsilon c)_{g_{1} \alpha_{1} A_{1}}^{\dagger} b_{f_{2} \alpha_{2} A_{2}}^{\dagger}(\epsilon c)_{f_{2} \alpha_{2} A_{2}}^{\dagger}\left|0_{F}\right\rangle$

(b) $b_{f_{1} \alpha_{1} A_{1}}^{\dagger}\left(\mathcal{T}_{F}\right)_{f_{1} g_{1}}\left(\tau_{i}\right)_{\alpha_{1} \beta_{1}}(\epsilon c)_{g_{1} \beta_{1} A_{1}}^{\dagger} b_{f_{2} \alpha_{2} A_{2}}^{\dagger}\left(\tau_{i}\right)_{\alpha_{2} \beta_{2}}(\epsilon c)_{f_{2} \beta_{2} A_{2}}^{\dagger}\left|0_{F}\right\rangle$

(c) $b_{f_{1} \alpha_{1} A_{1}}^{\dagger}\left(\mathcal{T}_{F}\right)_{f_{1} g_{1}}\left(T_{a}\right)_{A_{1} B_{1}}(\epsilon c)_{g_{1} \alpha_{1} B_{1}}^{\dagger} b_{f_{2} \alpha_{2} A_{2}}^{\dagger}\left(T_{a}\right)_{A_{2} B_{2}}(\epsilon c)_{f_{2} \alpha_{2} A_{2}}^{\dagger}\left|0_{F}\right\rangle$

(d) $b_{f_{1} \alpha_{1} A_{1}}^{\dagger}\left(\mathcal{T}_{F}\right)_{f_{1} g_{1}}\left(\tau_{i}\right)_{\alpha_{1} \beta_{1}}\left(T_{a}\right)_{A_{1} B_{1}}(\epsilon c)_{g_{1} \beta_{1} B_{1}}^{\dagger} b_{f_{2} \alpha_{2} A_{2}}^{\dagger}\left(\tau_{i}\right)_{\alpha_{2} \beta_{2}}\left(T_{a}\right)_{A_{2} B_{2}}(\epsilon c)_{f_{2} \beta_{2} B_{2}}^{\dagger}\left|0_{F}\right\rangle$

(11) $(\mathbf{8}, \mathbf{1}, \mathbf{1})$ :

(a) $b_{f_{1} \alpha_{1} A_{1}}^{\dagger}\left(\mathcal{T}_{F}\right)_{f_{1} g_{1}}\left(\tau_{i}\right)_{\alpha_{1} \beta_{1}}(\epsilon c)_{g_{1} \beta_{1} A_{1}}^{\dagger} b_{f_{2} \alpha_{2} A_{2}}^{\dagger}(\epsilon c)_{f_{2} \alpha_{2} A_{2}}^{\dagger}\left|0_{F}\right\rangle$

(b) $b_{f_{1} \alpha_{1} A_{1}}^{\dagger}\left(\mathcal{T}_{F}\right)_{f_{1} g_{1}}\left(\tau_{i}\right)_{\alpha_{1} \beta_{1}}\left(T_{a}\right)_{A_{1} B_{1}}(\epsilon c)_{g_{1} \beta_{1} B_{1}}^{\dagger} b_{f_{2} \alpha_{2} A_{2}}^{\dagger}\left(T_{a}\right)_{A_{2} B_{2}}(\epsilon c)_{f_{2} \alpha_{2} A_{2}}^{\dagger}\left|0_{F}\right\rangle$

(c) $b_{f_{1} \alpha_{1} A_{1}}^{\dagger}\left(\mathcal{T}_{F}\right)_{f_{1} g_{1}}(\epsilon c)_{g_{1} \alpha_{1} A_{1}}^{\dagger} b_{f_{2} \alpha_{2} A_{2}}^{\dagger}\left(\tau_{i}\right)_{\alpha_{2} \beta_{2}}(\epsilon c)_{f_{2} \beta_{2} A_{2}}^{\dagger}\left|0_{F}\right\rangle$

(d) $b_{f_{1} \alpha_{1} A_{1}}^{\dagger}\left(\mathcal{T}_{F}\right)_{f_{1} g_{1}}\left(T_{a}\right)_{A_{1} B_{1}}(\epsilon c)_{g_{1} \alpha_{1} B_{1}}^{\dagger} b_{f_{2} \alpha_{2} A_{2}}^{\dagger}\left(\tau_{i}\right)_{\alpha_{2} \beta_{2}}\left(T_{a}\right)_{A_{2} B_{2}}(\epsilon c)_{f_{2} \beta_{2} A_{2}}^{\dagger}\left|0_{F}\right\rangle$

(12) $(\mathbf{8}, \mathbf{2}, \mathbf{1})$ :

(a) $b_{f_{1} \alpha_{1} A_{1}}^{\dagger}\left(\mathcal{T}_{F}\right)_{f_{1} g_{1}}\left(\tau_{i}\right)_{\alpha_{1} \beta_{1}}(\epsilon c)_{g_{1} \beta_{1} A_{1}}^{\dagger} b_{f_{2} \alpha_{2} A_{2}}^{\dagger}\left(\tau_{j}\right)_{\alpha_{2} \beta_{2}}(\epsilon c)_{f_{2} \beta_{2} A_{2}}^{\dagger}\left|0_{F}\right\rangle$

(b) $b_{f_{1} \alpha_{1} A_{1}}^{\dagger}\left(\mathcal{T}_{F}\right)_{f_{1} g_{1}}\left(\tau_{i}\right)_{\alpha_{1} \beta_{1}}\left(T_{a}\right)_{A_{1} B_{1}}(\epsilon c)_{g_{1} \beta_{1} B_{1}}^{\dagger} b_{f_{2} \alpha_{2} A_{2}}^{\dagger}\left(\tau_{j}\right)_{\alpha_{2} \beta_{2}}\left(T_{a}\right)_{A_{2} B_{2}}(\epsilon c)_{f_{2} \beta_{2} A_{2}}^{\dagger}\left|0_{F}\right\rangle$

(13) $(\mathbf{8 , 0 , 8})$ :
(a) $b_{f_{1} \alpha_{1} A_{1}}^{\dagger}\left(\mathcal{T}_{F}\right)_{f_{1} g_{1}}\left(T_{a}\right)_{A_{1} B_{1}}(\epsilon c)_{g_{1} \alpha_{1} B_{1}}^{\dagger} b_{f_{2} \alpha_{2} A_{2}}^{\dagger}(\epsilon c)_{f_{2} \alpha_{2} A_{2}}^{\dagger}\left|0_{F}\right\rangle$
(b) $b_{f_{1} \alpha_{1} A_{1}}^{\dagger}\left(\mathcal{T}_{F}\right)_{f_{1} g_{1}}\left(\tau_{i}\right)_{\alpha_{1} \beta_{1}}\left(T_{a}\right)_{A_{1} B_{1}}(\epsilon c)_{g_{1} \beta_{1} B_{1}}^{\dagger} b_{f_{2} \alpha_{2} A_{2}}^{\dagger}\left(\tau_{i}\right)_{\alpha_{2} \beta_{2}}(\epsilon c)_{f_{2} \beta_{2} A_{2}}^{\dagger}\left|0_{F}\right\rangle$
(c) $b_{f_{1} \alpha_{1} A_{1}}^{\dagger}\left(\mathcal{T}_{F}\right)_{f_{1} g_{1}}(\epsilon c)_{g_{1} \alpha_{1} A_{1}}^{\dagger} b_{f_{2} \alpha_{2} A_{2}}^{\dagger}\left(T_{a}\right)_{A_{2} B_{2}}(\epsilon c)_{f_{2} \alpha_{2} B_{2}}^{\dagger}\left|0_{F}\right\rangle$ 
(d) $b_{f_{1} \alpha_{1} A_{1}}^{\dagger}\left(\mathcal{T}_{F}\right)_{f_{1} g_{1}}\left(\tau_{i}\right)_{\alpha_{1} \beta_{1}}(\epsilon c)_{g_{1} \beta_{1} A_{1}}^{\dagger} b_{f_{2} \alpha_{2} A_{2}}^{\dagger}\left(\tau_{i}\right)_{\alpha_{2} \beta_{2}}\left(T_{a}\right)_{A_{2} B_{2}}(\epsilon c)_{f_{2} \beta_{2} B_{2}}^{\dagger}\left|O_{F}\right\rangle$

(e) $f_{a b c} b_{f_{1} \alpha_{1} A_{1}}^{\dagger}\left(\mathcal{T}_{F}\right)_{f_{1} g_{1}}\left(T_{b}\right)_{A_{1} B_{1}}(\epsilon c)_{g_{1} \alpha_{1} B_{1}}^{\dagger} b_{f_{2} \alpha_{2} A_{2}}^{\dagger}\left(T_{c}\right)_{A_{2} B_{2}}(\epsilon c)_{f_{2} \alpha_{2} B_{2}}^{\dagger}\left|0_{F}\right\rangle$

(f) $d_{a b c} b_{f_{1} \alpha_{1} A_{1}}^{\dagger}\left(\mathcal{I}_{F}\right)_{f_{1} g_{1}}\left(T_{b}\right)_{A_{1} B_{1}}(\epsilon c)_{g_{1} \alpha_{1} B_{1}}^{\dagger} b_{f_{2} \alpha_{2} A_{2}}^{\dagger}\left(T_{c}\right)_{A_{2} B_{2}}(\epsilon c)_{f_{2} \alpha_{2} B_{2}}^{\dagger}\left|0_{F}\right\rangle$

(g) $f_{a b c} b_{f_{1} \alpha_{1} A_{1}}^{\dagger}\left(\mathcal{T}_{F}\right)_{f_{1} g_{1}}\left(\tau_{i}\right)_{\alpha_{1} \beta_{1}}\left(T_{b}\right)_{A_{1} B_{1}}(\epsilon c)_{g_{1} \beta_{1} B_{1}}^{\dagger} b_{f_{2} \alpha_{2} A_{2}}^{\dagger}\left(\tau_{i}\right)_{\alpha_{2} \beta_{2}}\left(T_{c}\right)_{A_{2} B_{2}}(\epsilon c)_{f_{2} \beta_{2} B_{2}}^{\dagger}\left|0_{F}\right\rangle$

(h) $d_{a b c} b_{f_{1} \alpha_{1} A_{1}}^{\dagger}\left(\mathcal{T}_{F}\right)_{f_{1} g_{1}}\left(\tau_{i}\right)_{\alpha_{1} \beta_{1}}\left(T_{b}\right)_{A_{1} B_{1}}(\epsilon c)_{g_{1} \beta_{1} B_{1}}^{\dagger} b_{f_{2} \alpha_{2} A_{2}}^{\dagger}\left(\tau_{i}\right)_{\alpha_{2} \beta_{2}}\left(T_{c}\right)_{A_{2} B_{2}}(\epsilon c)_{f_{2} \beta_{2} B_{2}}^{\dagger}\left|0_{F}\right\rangle$

(14) $(\mathbf{8}, \mathbf{1}, \mathbf{8})$ :
(a) $b_{f_{1} \alpha_{1} A_{1}}^{\dagger}\left(\mathcal{T}_{F}\right)_{f_{1} g_{1}}\left(\tau_{i}\right)_{\alpha_{1} \beta_{1}}\left(T_{a}\right)_{A_{1} B_{1}}(\epsilon c)_{g_{1} \beta_{1} B_{1}}^{\dagger} b_{f_{2} \alpha_{2} A_{2}}^{\dagger}(\epsilon c)_{f_{2} \alpha_{2} A_{2}}^{\dagger}\left|0_{F}\right\rangle$
(b) $b_{f_{1} \alpha_{1} A_{1}}^{\dagger}\left(\mathcal{T}_{F}\right)_{f_{1} g_{1}}\left(\tau_{i}\right)_{\alpha_{1} \beta_{1}}(\epsilon c)_{g_{1} \beta_{1} A_{1}}^{\dagger} b_{f_{2} \alpha_{2} A_{2}}^{\dagger}\left(T_{a}\right)_{A_{2} B_{2}}(\epsilon c)_{f_{2} \alpha_{2} B_{2}}^{\dagger}\left|0_{F}\right\rangle$
(c) $b_{f_{1} \alpha_{1} A_{1}}^{\dagger}\left(\mathcal{T}_{F}\right)_{f_{1} g_{1}}\left(T_{a}\right)_{A_{1} B_{1}}(\epsilon c)_{g_{1} \alpha_{1} B_{1}}^{\dagger} b_{f_{2} \alpha_{2} A_{2}}^{\dagger}\left(\tau_{i}\right)_{\alpha_{2} \beta_{2}}(\epsilon c)_{f_{2} \beta_{2} A_{2}}^{\dagger}\left|0_{F}\right\rangle$
(d) $b_{f_{1} \alpha_{1} A_{1}}^{\dagger}\left(\mathcal{T}_{F}\right)_{f_{1} g_{1}}(\epsilon c)_{g_{1} \alpha_{1} A_{1}}^{\dagger} b_{f_{2} \alpha_{2} A_{2}}^{\dagger}\left(\tau_{i}\right)_{\alpha_{2} \beta_{2}}\left(T_{a}\right)_{A_{2} B_{2}}(\epsilon c)_{f_{2} \beta_{2} B_{2}}^{\dagger}\left|0_{F}\right\rangle$
(e) $f_{a b c} b_{f_{1} \alpha_{1} A_{1}}^{\dagger}\left(\mathcal{T}_{F}\right)_{f_{1} g_{1}}\left(\tau_{i}\right)_{\alpha_{1} \beta_{1}}\left(T_{b}\right)_{A_{1} B_{1}}(\epsilon c)_{g_{1} \beta_{1} B_{1}}^{\dagger} b_{f_{2} \alpha_{2} A_{2}}^{\dagger}\left(T_{c}\right)_{A_{2} B_{2}}(\epsilon c)_{f_{2} \alpha_{2} A_{2}}^{\dagger}\left|0_{F}\right\rangle$
(f) $d_{a b c} b_{f_{1} \alpha_{1} A_{1}}^{\dagger}\left(\mathcal{T}_{F}\right)_{f_{1} g_{1}}\left(\tau_{i}\right)_{\alpha_{1} \beta_{1}}\left(T_{b}\right)_{A_{1} B_{1}}(\epsilon c)_{g_{1} \beta_{1} B_{1}}^{\dagger} b_{f_{2} \alpha_{2} A_{2}}^{\dagger}\left(T_{c}\right)_{A_{2} B_{2}}(\epsilon c)_{f_{2} \alpha_{2} A_{2}}^{\dagger}\left|0_{F}\right\rangle$
(g) $f_{a b c} b_{f_{1} \alpha_{1} A_{1}}^{\dagger}\left(\mathcal{T}_{F}\right)_{f_{1} g_{1}}\left(T_{b}\right)_{A_{1} B_{1}}(\epsilon c)_{g_{1} \alpha_{1} B_{1}}^{\dagger} b_{f_{2} \alpha_{2} A_{2}}^{\dagger}\left(\tau_{i}\right)_{\alpha_{2} \beta_{2}}\left(T_{c}\right)_{A_{2} B_{2}}(\epsilon c)_{f_{2} \beta_{2} A_{2}}^{\dagger}\left|0_{F}\right\rangle$
(h) $d_{a b c} b_{f_{1} \alpha_{1} A_{1}}^{\dagger}\left(\mathcal{T}_{F}\right)_{f_{1} g_{1}}\left(T_{b}\right)_{A_{1} B_{1}}(\epsilon c)_{g_{1} \alpha_{1} B_{1}}^{\dagger} b_{f_{2} \alpha_{2} A_{2}}^{\dagger}\left(\tau_{i}\right)_{\alpha_{2} \beta_{2}}\left(T_{c}\right)_{A_{2} B_{2}}(\epsilon c)_{f_{2} \beta_{2} A_{2}}^{\dagger}\left|0_{F}\right\rangle$
(i) $\epsilon_{i j k} b_{f_{1} \alpha_{1} A_{1}}^{\dagger}\left(\mathcal{T}_{F}\right)_{f_{1} g_{1}}\left(\tau_{j}\right)_{\alpha_{1} \beta_{1}}\left(T_{a}\right)_{A_{1} B_{1}}(\epsilon c)_{g_{1} \beta_{1} B_{1}}^{\dagger} b_{f_{2} \alpha_{2} A_{2}}^{\dagger}\left(\tau_{k}\right)_{\alpha_{2} \beta_{2}}(\epsilon c)_{f_{2} \beta_{2} A_{2}}^{\dagger}\left|0_{F}\right\rangle$
(j) $\epsilon_{i j k} b_{f_{1} \alpha_{1} A_{1}}^{\dagger}\left(\mathcal{T}_{F}\right)_{f_{1} g_{1}}\left(\tau_{j}\right)_{\alpha_{1} \beta_{1}}(\epsilon c)_{g_{1} \beta_{1} A_{1}}^{\dagger} b_{f_{2} \alpha_{2} A_{2}}^{\dagger}\left(\tau_{k}\right)_{\alpha_{2} \beta_{2}}\left(T_{a}\right)_{A_{2} B_{2}}(\epsilon c)_{f_{2} \beta_{2} B_{2}}^{\dagger}\left|0_{F}\right\rangle$
(k) $\epsilon_{i j k} f_{a b c} b_{f_{1} \alpha_{1} A_{1}}^{\dagger}\left(\mathcal{T}_{F}\right)_{f_{1} g_{1}}\left(\tau_{j}\right)_{\alpha_{1} \beta_{1}}\left(T_{b}\right)_{A_{1} B_{1}}(\epsilon c)_{g_{1} \beta_{1} B_{1}}^{\dagger} b_{f_{2} \alpha_{2} A_{2}}^{\dagger}\left(\tau_{k}\right)_{\alpha_{2} \beta_{2}}\left(T_{c}\right)_{A_{2} B_{2}}(\epsilon c)_{f_{2} \beta_{2} B_{2}}^{\dagger}\left|0_{F}\right\rangle$
(l) $\epsilon_{i j k} d_{a b c} b_{f_{1} \alpha_{1} A_{1}}^{\dagger}\left(\mathcal{T}_{F}\right)_{f_{1} g_{1}}\left(\tau_{j}\right)_{\alpha_{1} \beta_{1}}\left(T_{b}\right)_{A_{1} B_{1}}(\epsilon c)_{g_{1} \beta_{1} B_{1}}^{\dagger} b_{f_{2} \alpha_{2} A_{2}}^{\dagger}\left(\tau_{k}\right)_{\alpha_{2} \beta_{2}}\left(T_{c}\right)_{A_{2} B_{2}}(\epsilon c)_{f_{2} \beta_{2} B_{2}}^{\dagger}\left|0_{F}\right\rangle$

(15) $(\mathbf{8}, \mathbf{2}, \mathbf{8})$ :
(a) $b_{f_{1} \alpha_{1} A_{1}}^{\dagger}\left(\mathcal{T}_{F}\right)_{f_{1} g_{1}}\left(\tau_{i}\right)_{\alpha_{1} \beta_{1}}\left(T_{a}\right)_{A_{1} B_{1}}(\epsilon c)_{g_{1} \beta_{1} B_{1}}^{\dagger} b_{f_{2} \alpha_{2} A_{2}}^{\dagger}\left(\tau_{j}\right)_{\alpha_{2} \beta_{2}}(\epsilon c)_{f_{2} \beta_{2} A_{2}}^{\dagger}\left|0_{F}\right\rangle$
(b) $b_{f_{1} \alpha_{1} A_{1}}^{\dagger}\left(\mathcal{T}_{F}\right)_{f_{1} g_{1}}\left(\tau_{i}\right)_{\alpha_{1} \beta_{1}}(\epsilon c)_{g_{1} \beta_{1} A_{1}}^{\dagger} b_{f_{2} \alpha_{2} A_{2}}^{\dagger}\left(\tau_{j}\right)_{\alpha_{2} \beta_{2}}\left(T_{a}\right)_{A_{2} B_{2}}(\epsilon c)_{f_{2} \beta_{2} B_{2}}^{\dagger}\left|0_{F}\right\rangle$
(c) $f_{a b c} b_{f_{1} \alpha_{1} A_{1}}^{\dagger}\left(\mathcal{T}_{F}\right)_{f_{1} g_{1}}\left(\tau_{i}\right)_{\alpha_{1} \beta_{1}}\left(T_{b}\right)_{A_{1} B_{1}}(\epsilon c)_{g_{1} \beta_{1} B_{1}}^{\dagger} b_{f_{2} \alpha_{2} A_{2}}^{\dagger}\left(\tau_{j}\right)_{\alpha_{2} \beta_{2}}\left(T_{c}\right)_{A_{2} B_{2}}(\epsilon c)_{f_{2} \beta_{2} A_{2}}^{\dagger}\left|0_{F}\right\rangle$
(d) $d_{a b c} b_{f_{1} \alpha_{1} A_{1}}^{\dagger}\left(\mathcal{T}_{F}\right)_{f_{1} g_{1}}\left(\tau_{i}\right)_{\alpha_{1} \beta_{1}}\left(T_{b}\right)_{A_{1} B_{1}}(\epsilon c)_{g_{1} \beta_{1} B_{1}}^{\dagger} b_{f_{2} \alpha_{2} A_{2}}^{\dagger}\left(\tau_{j}\right)_{\alpha_{2} \beta_{2}}\left(T_{c}\right)_{A_{2} B_{2}}(\epsilon c)_{f_{2} \beta_{2} A_{2}}^{\dagger}\left|0_{F}\right\rangle$

(16) $(\mathbf{8}, \mathbf{0}, 27 \oplus \mathbf{2 0})$ :

(a) $b_{f_{1} \alpha_{1} A_{1}}^{\dagger}\left(\mathcal{T}_{F}\right)_{f_{1} g_{1}}\left(T_{a}\right)_{A_{1} B_{1}}(\epsilon c)_{g_{1} \alpha_{1} B_{1}}^{\dagger} b_{f_{2} \alpha_{2} A_{2}}^{\dagger}\left(T_{b}\right)_{A_{2} B_{2}}(\epsilon c)_{f_{2} \alpha_{2} B_{2}}^{\dagger}\left|0_{F}\right\rangle$

(b) $b_{f_{1} \alpha_{1} A_{1}}^{\dagger}\left(\mathcal{T}_{F}\right)_{f_{1} g_{1}}\left(\tau_{i}\right)_{\alpha_{1} \beta_{1}}\left(T_{a}\right)_{A_{1} B_{1}}(\epsilon c)_{g_{1} \beta_{1} A_{1}}^{\dagger} b_{f_{2} \alpha_{2} A_{2}}^{\dagger}\left(\tau_{i}\right)_{\alpha_{2} \beta_{2}}\left(T_{b}\right)_{A_{1} B_{2}}(\epsilon c)_{f_{2} \beta_{2} B_{2}}^{\dagger}\left|0_{F}\right\rangle$

(17) $(\mathbf{8}, \mathbf{1}, \mathbf{2 7} \oplus \mathbf{2 0})$ :
(a) $b_{f_{1} \alpha_{1} A_{1}}^{\dagger}\left(\mathcal{T}_{F}\right)_{f_{1} g_{1}}\left(\tau_{i}\right)_{\alpha_{1} \beta_{1}}\left(T_{a}\right)_{A_{1} B_{1}}(\epsilon c)_{g_{1} \beta_{1} B_{1}}^{\dagger} b_{f_{2} \alpha_{2} A_{2}}^{\dagger}\left(T_{b}\right)_{A_{2} B_{2}}(\epsilon c)_{f_{2} \alpha_{2} B_{2}}^{\dagger}\left|0_{F}\right\rangle$
(b) $\epsilon_{i j k} b_{f_{1} \alpha_{1} A_{1}}^{\dagger}\left(\mathcal{T}_{F}\right)_{f_{1} g_{1}}\left(\tau_{j}\right)_{\alpha_{1} \beta_{1}}\left(T_{a}\right)_{A_{1} B_{1}}(\epsilon c)_{g_{1} \beta_{1} A_{1}}^{\dagger} b_{f_{2} \alpha_{2} A_{2}}^{\dagger}\left(\tau_{k}\right)_{\alpha_{2} \beta_{2}}\left(T_{b}\right)_{A_{1} B_{2}}(\epsilon c)_{f_{2} \beta_{2} B_{2}}^{\dagger}\left|0_{F}\right\rangle$

(18) $(\mathbf{8}, \mathbf{2}, 27 \oplus \mathbf{2 0})$ :

(a) $b_{f_{1} \alpha_{1} A_{1}}^{\dagger}\left(\mathcal{T}_{F}\right)_{f_{1} g_{1}}\left(\tau_{i}\right)_{\alpha_{1} \beta_{1}}\left(T_{a}\right)_{A_{1} B_{1}}(\epsilon c)_{g_{1} \beta_{1} A_{1}}^{\dagger} b_{f_{2} \alpha_{2} A_{2}}^{\dagger}\left(\tau_{j}\right)_{\alpha_{2} \beta_{2}}\left(T_{b}\right)_{A_{1} B_{2}}(\epsilon c)_{f_{2} \beta_{2} B_{2}}^{\dagger}\left|0_{F}\right\rangle$

Baryon and baryon-meson states are organized into an octet and a decuplet under flavor $S U(3)$. We define $\left(T_{F}\right)_{f g h} \equiv\left(\mathcal{T}_{F}\right)_{f f_{1}} \epsilon_{f_{1} g h}, f, g, h=1, \ldots, 3$ and $F=1, \ldots 8$. We also define the tensor $\Delta_{f, g, h}^{\rho}, f, g, h=1, \ldots, 3$ and $\rho=$ $1, \ldots, 10$ such that $\Delta$ is totally symmetric in $f, g, h$ and generates the flavor decuplet. States that carry both adjoint spin indices $i, j, \ldots$ and a fundamental spin index $\gamma$ can be separated into different spin components using suitable projectors; however, it is convenient to express them like this before combining them with gluon wave functions. It is also convenient to define $\left(\mathcal{E}_{A}\right)_{B C} \equiv \epsilon_{A B C}$ for fundamental indices $A, B, C=1$ to 3 .

\section{c. Baryon states}

(1) $\left(\mathbf{8}, \frac{\mathbf{1}}{\mathbf{2}}, \mathbf{1}\right):\left(T_{F}\right)_{f g h}\left(\mathcal{E}_{A}\right)_{B C} \epsilon_{\alpha \beta} b_{f \alpha A}^{\dagger} b_{g \beta B}^{\dagger} b_{h \gamma C}^{\dagger}\left|0_{F}\right\rangle$

(2) $\left(\mathbf{8}, \mathbf{1} \otimes \frac{1}{\mathbf{2}}, \mathbf{1}\right):\left(T_{F}\right)_{f g h}\left(\mathcal{E}_{A}\right)_{B C}\left(\tau_{i} \epsilon\right)_{\alpha \beta} b_{f \alpha A}^{\dagger} b_{g \beta B}^{\dagger} b_{h \gamma C}^{\dagger}\left|0_{F}\right\rangle$

(3) $\left(\mathbf{8}, \frac{1}{2}, \mathbf{8}\right):\left(T_{F}\right)_{f g h}\left(\mathcal{E}_{A} T_{a}\right)_{B C} \epsilon_{\alpha \beta} b_{f \alpha A}^{\dagger} b_{g \beta B}^{\dagger} b_{h \gamma C}^{\dagger}\left|0_{F}\right\rangle$

(4) $\left(\mathbf{8}, \mathbf{1} \otimes \frac{1}{2}, \mathbf{8}\right):\left(T_{F}\right)_{f g h}\left(\mathcal{E}_{A} T_{a}\right)_{B C}\left(\tau_{i} \epsilon\right)_{\alpha \beta} b_{f \alpha A}^{\dagger} b_{g \beta B}^{\dagger} b_{h \gamma C}^{\dagger}\left|0_{F}\right\rangle$

(5) $\left(\mathbf{1 0}, \mathbf{1} \otimes \frac{1}{2}, \mathbf{1}\right): \Delta_{f g h}^{\rho}\left(\mathcal{E}_{A}\right)_{B C}\left(\tau_{i} \epsilon\right)_{\alpha \beta} b_{f \alpha A}^{\dagger} b_{g \beta B}^{\dagger} b_{h \gamma C}^{\dagger}\left|0_{F}\right\rangle$

(6) $\left(\mathbf{1 0}, \frac{1}{2}, \mathbf{8}\right): \Delta_{f g h}^{\rho}\left(\mathcal{E}_{A} T_{a}\right)_{B C} \epsilon_{\alpha \beta} b_{f \alpha A}^{\dagger} b_{g \beta B}^{\dagger} b_{h \gamma C}^{\dagger}\left|0_{F}\right\rangle$ 
(7) $\left(\mathbf{1 0}, \mathbf{1} \otimes \frac{\mathbf{1}}{\mathbf{2}}, \mathbf{8}\right): \Delta_{f g h}^{\rho}\left(\mathcal{E}_{A} T_{a}\right)_{B C}\left(\tau_{i} \epsilon\right)_{\alpha \beta} b_{f \alpha A}^{\dagger} b_{g \beta B}^{\dagger} b_{h \gamma C}^{\dagger}\left|0_{F}\right\rangle$

Note: $\left(\mathbf{1 0}, \frac{\mathbf{1}}{\mathbf{2}}, \mathbf{1}\right)$ does not exist due to symmetry.

\section{d. Baryon-meson states}

(1) $\left(\mathbf{8}, \frac{1}{2}, \mathbf{1}\right)$ :

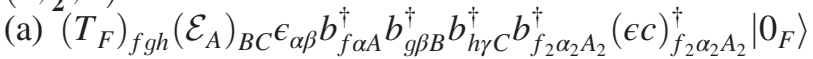

(b) $\left(T_{F}\right)_{f g h}\left(\mathcal{E}_{A}\right)_{B C}\left(\tau_{i} \epsilon\right)_{\alpha \beta} b_{f \alpha A}^{\dagger} b_{g \beta B}^{\dagger} b_{h \gamma C}^{\dagger} b_{f_{2} \alpha_{2} A_{2}}^{\dagger}\left(\tau_{i}\right)_{\alpha_{2} \beta_{2}}(\epsilon c)_{f_{2} \beta_{2} A_{2}}^{\dagger}\left|0_{F}\right\rangle$

(c) $\left(T_{F}\right)_{f g h}\left(\mathcal{E}_{A} T_{a}\right)_{B C} \epsilon_{\alpha \beta} b_{f \alpha A}^{\dagger} b_{g \beta B}^{\dagger} b_{h \gamma C}^{\dagger} b_{f_{2} \alpha_{2} A_{2}}^{\dagger}\left(T_{a}\right)_{A_{2} B_{2}}(\epsilon c)_{f_{2} \alpha_{2} A_{2}}^{\dagger}\left|0_{F}\right\rangle$

(d) $\left(T_{F}\right)_{f g h}\left(\mathcal{E}_{A} T_{a}\right)_{B C}\left(\tau_{i} \epsilon\right)_{\alpha \beta} b_{f \alpha A}^{\dagger} b_{g \beta B}^{\dagger} b_{h \gamma C}^{\dagger} b_{f_{2} \alpha_{2} A_{2}}^{\dagger}\left(\tau_{i}\right)_{\alpha_{2} \beta_{2}}\left(T_{a}\right)_{A_{2} B_{2}}(\epsilon c)_{f_{2} \beta_{2} B_{2}}^{\dagger}\left|0_{F}\right\rangle$

(2) $\left(\mathbf{8}, \mathbf{1} \otimes \frac{1}{2}, \mathbf{1}\right):$

(a) $\left(T_{F}\right)_{f g h}\left(\mathcal{E}_{A}\right)_{B C}\left(\tau_{i} \epsilon\right)_{\alpha \beta} b_{f \alpha A}^{\dagger} b_{g \beta B}^{\dagger} b_{h \gamma C}^{\dagger} b_{f_{2} \alpha_{2} A_{2}}^{\dagger}(\epsilon c)_{f_{2} \alpha_{2} A_{2}}^{\dagger}\left|0_{F}\right\rangle$

(b) $\left(T_{F}\right)_{f g h}\left(\mathcal{E}_{A} T_{a}\right)_{B C} \epsilon_{\alpha \beta} b_{f \alpha A}^{\dagger} b_{g \beta B}^{\dagger} b_{h \gamma C}^{\dagger} b_{f_{2} \alpha_{2} A_{2}}^{\dagger}\left(\tau_{i}\right)_{\alpha_{2} \beta_{2}}\left(T_{a}\right)_{A_{2} B_{2}}(\epsilon c)_{f_{2} \beta_{2} B_{2}}^{\dagger}\left|0_{F}\right\rangle$

(c) $\left(T_{F}\right)_{f g h}\left(\mathcal{E}_{A} T_{a}\right)_{B C}\left(\tau_{i} \epsilon\right)_{\alpha \beta} b_{f \alpha A}^{\dagger} b_{g \beta B}^{\dagger} b_{h \gamma C}^{\dagger} b_{f_{2} \alpha_{2} A_{2}}^{\dagger}\left(T_{a}\right)_{A_{2} B_{2}}(\epsilon c)_{f_{2} \alpha_{2} B_{2}}^{\dagger}\left|0_{F}\right\rangle$

(d) $\epsilon_{i j k}\left(T_{F}\right)_{f g h}\left(\mathcal{E}_{A}\right)_{B C}\left(\tau_{j} \epsilon\right)_{\alpha \beta} b_{f \alpha A}^{\dagger} b_{g \beta B}^{\dagger} b_{h \gamma C}^{\dagger} b_{f_{2} \alpha_{2} A_{2}}^{\dagger}\left(\tau_{k}\right)_{\alpha_{2} \beta_{2}}(\epsilon c)_{f_{2} \beta_{2} A_{2}}^{\dagger}\left|0_{F}\right\rangle$

(e) $\epsilon_{i j k}\left(T_{F}\right)_{f g h}\left(\mathcal{E}_{A} T_{a}\right)_{B C}\left(\tau_{j} \epsilon\right)_{\alpha \beta} b_{f \alpha A}^{\dagger} b_{g \beta B}^{\dagger} b_{h \gamma C}^{\dagger} b_{f_{2} \alpha_{2} A_{2}}^{\dagger}\left(\tau_{k}\right)_{\alpha_{2} \beta_{2}}\left(T_{a}\right)_{A_{2} B_{2}}(\epsilon c)_{f_{2} \beta_{2} B_{2}}^{\dagger}\left|0_{F}\right\rangle$

(f) $\left(T_{F}\right)_{f g h}\left(\mathcal{E}_{A}\right)_{B C} \epsilon_{\alpha \beta} b_{f \alpha A}^{\dagger} b_{g \beta B}^{\dagger} b_{h \gamma C}^{\dagger} b_{f_{2} \alpha_{2} A_{2}}^{\dagger}\left(\tau_{i}\right)_{\alpha_{2} \beta_{2}}(\epsilon c)_{f_{2} \beta_{2} B_{2}}^{\dagger}\left|0_{F}\right\rangle$

(3) $\left(\mathbf{8}, \mathbf{2} \otimes \frac{1}{2}, \mathbf{1}\right)$ :

(a) $\left(T_{F}\right)_{f g h}\left(\mathcal{E}_{A}\right)_{B C}\left(\tau_{i} \epsilon\right)_{\alpha \beta} b_{f \alpha A}^{\dagger} b_{g \beta B}^{\dagger} b_{h \gamma C}^{\dagger} b_{f_{2} \alpha_{2} A_{2}}^{\dagger}\left(\tau_{j}\right)_{\alpha_{2} \beta_{2}}(\epsilon c)_{f_{2} \beta_{2} A_{2}}^{\dagger}\left|0_{F}\right\rangle$

(b) $\left(T_{F}\right)_{f g h}\left(\mathcal{E}_{A} T_{a}\right)_{B C}\left(\tau_{i} \epsilon\right)_{\alpha \beta} b_{f \alpha A}^{\dagger} b_{g \beta B}^{\dagger} b_{h \gamma C}^{\dagger} b_{f_{2} \alpha_{2} A_{2}}^{\dagger}\left(\tau_{j}\right)_{\alpha_{2} \beta_{2}}\left(T_{a}\right)_{A_{2} B_{2}}(\epsilon c)_{f_{2} \beta_{2} A_{2}}^{\dagger}\left|0_{F}\right\rangle$

(4) $\left(\mathbf{8}, \frac{1}{2}, 8\right)$ :

(a) $\left(T_{F}\right)_{f g h}\left(\mathcal{E}_{A}\right)_{B C} \epsilon_{\alpha \beta} b_{f \alpha A}^{\dagger} b_{g \beta B}^{\dagger} b_{h \gamma C}^{\dagger} b_{f_{2} \alpha_{2} A_{2}}^{\dagger}\left(T_{a}\right)_{A_{2} B_{2}}(\epsilon c)_{f_{2} \alpha_{2} B_{2}}^{\dagger}\left|0_{F}\right\rangle$

(b) $\left(T_{F}\right)_{f g h}\left(\mathcal{E}_{A} T_{a}\right)_{B C} \epsilon_{\alpha \beta} b_{f \alpha A}^{\dagger} b_{g \beta B}^{\dagger} b_{h \gamma C}^{\dagger} b_{f_{2} \alpha_{2} A_{2}}^{\dagger}(\epsilon c)_{f_{2} \alpha_{2} A_{2}}^{\dagger}\left|O_{F}\right\rangle$

(c) $\left(T_{F}\right)_{f g h}\left(\mathcal{E}_{A}\right)_{B C}\left(\tau_{i} \epsilon\right)_{\alpha \beta} b_{f \alpha A}^{\dagger} b_{g \beta B}^{\dagger} b_{h \gamma C}^{\dagger} b_{f_{2} \alpha_{2} A_{2}}^{\dagger}\left(\tau_{i}\right)_{\alpha_{2} \beta_{2}}\left(T_{a}\right)_{A_{2} B_{2}}(\epsilon c)_{f_{2} \beta_{2} B_{2}}^{\dagger}\left|O_{F}\right\rangle$

(d) $\left(T_{F}\right)_{f g h}\left(\mathcal{E}_{A} T_{a}\right)_{B C}\left(\tau_{i} \epsilon\right)_{\alpha \beta} b_{f \alpha A}^{\dagger} b_{g \beta B}^{\dagger} b_{h \gamma C}^{\dagger} b_{f_{2} \alpha_{2} A_{2}}^{\dagger}\left(\tau_{i}\right)_{\alpha_{2} \beta_{2}}(\epsilon c)_{f_{2} \beta_{2} A_{2}}^{\dagger}\left|0_{F}\right\rangle$

(e) $f_{a b c}\left(T_{F}\right)_{f g h}\left(\mathcal{E}_{A} T_{b}\right)_{B C} \epsilon_{\alpha \beta} b_{f \alpha A}^{\dagger} b_{g \beta B}^{\dagger} b_{h \gamma C}^{\dagger} b_{f_{2} \alpha_{2} A_{2}}^{\dagger}\left(T_{c}\right)_{A_{2} B_{2}}(\epsilon c)_{f_{2} \alpha_{2} B_{2}}^{\dagger}\left|0_{F}\right\rangle$

(f) $d_{a b c}\left(T_{F}\right)_{f g h}\left(\mathcal{E}_{A} T_{b}\right)_{B C} \epsilon_{\alpha \beta} b_{f \alpha A}^{\dagger} b_{g \beta B}^{\dagger} b_{h \gamma C}^{\dagger} b_{f_{2} \alpha_{2} A_{2}}^{\dagger}\left(T_{c}\right)_{A_{2} B_{2}}(\epsilon c)_{f_{2} \alpha_{2} B_{2}}^{\dagger}\left|0_{F}\right\rangle$

(g) $f_{a b c}\left(T_{F}\right)_{f g h}\left(\mathcal{E}_{A} T_{b}\right)_{B C}\left(\tau_{i} \epsilon\right)_{\alpha \beta} b_{f \alpha A}^{\dagger} b_{g \beta B}^{\dagger} b_{h \gamma C}^{\dagger} b_{f_{2} \alpha_{2} A_{2}}^{\dagger}\left(\tau_{i}\right)_{\alpha_{2} \beta_{2}}\left(T_{c}\right)_{A_{2} B_{2}}(\epsilon c)_{f_{2} \beta_{2} B_{2}}^{\dagger}\left|0_{F}\right\rangle$

(h) $d_{a b c}\left(T_{F}\right)_{f g h}\left(\mathcal{E}_{A} T_{b}\right)_{B C}\left(\tau_{i} \epsilon\right)_{\alpha \beta} b_{f \alpha A}^{\dagger} b_{g \beta B}^{\dagger} b_{h \gamma C}^{\dagger} b_{f_{2} \alpha_{2} A_{2}}^{\dagger}\left(\tau_{i}\right)_{\alpha_{2} \beta_{2}}\left(T_{c}\right)_{A_{2} B_{2}}(\epsilon c)_{f_{2} \beta_{2} B_{2}}^{\dagger}\left|0_{F}\right\rangle$

(5) $\left(\mathbf{8}, \mathbf{1} \otimes \frac{1}{2}, \mathbf{8}\right)$ :
(a) $\left(T_{F}\right)_{f g h}\left(\mathcal{E}_{A} T_{a}\right)_{B C}\left(\tau_{i} \epsilon\right)_{\alpha \beta} b_{f \alpha A}^{\dagger} b_{g \beta B}^{\dagger} b_{h \gamma C}^{\dagger} b_{f_{2} \alpha_{2} A_{2}}^{\dagger}(\epsilon c)_{f_{2} \alpha_{2} A_{2}}^{\dagger}\left|0_{F}\right\rangle$
(b) $\left(T_{F}\right)_{f g h}\left(\mathcal{E}_{A}\right)_{B C}\left(\tau_{i} \epsilon\right)_{\alpha \beta} b_{f \alpha A}^{\dagger} b_{g \beta B}^{\dagger} b_{h \gamma C}^{\dagger} b_{f_{2} \alpha_{2} A_{2}}^{\dagger}\left(T_{a}\right)_{A_{2} B_{2}}(\epsilon c)_{f_{2} \alpha_{2} B_{2}}^{\dagger}\left|0_{F}\right\rangle$
(c) $\left(T_{F}\right)_{f g h}\left(\mathcal{E}_{A} T_{a}\right)_{B C} \epsilon_{\alpha \beta} b_{f \alpha A}^{\dagger} b_{g \beta B}^{\dagger} b_{h \gamma C}^{\dagger} b_{f_{2} \alpha_{2} A_{2}}^{\dagger}\left(\tau_{i}\right)_{\alpha_{2} \beta_{2}}(\epsilon c)_{f_{2} \beta_{2} A_{2}}^{\dagger}\left|0_{F}\right\rangle$
(d) $\left(T_{F}\right)_{f g h}\left(\mathcal{E}_{A}\right)_{B C} \epsilon_{\alpha \beta} b_{f \alpha A}^{\dagger} b_{g \beta B}^{\dagger} b_{h \gamma C}^{\dagger} b_{f_{2} \alpha_{2} A_{2}}^{\dagger}\left(\tau_{i}\right)_{\alpha_{2} \beta_{2}}\left(T_{a}\right)_{A_{2} B_{2}}(\epsilon c)_{f_{2} \beta_{2} B_{2}}^{\dagger}\left|0_{F}\right\rangle$
(e) $f_{a b c}\left(T_{F}\right)_{f g h}\left(\mathcal{E}_{A} T_{b}\right)_{B C}\left(\tau_{i} \epsilon\right)_{\alpha \beta} b_{f \alpha A}^{\dagger} b_{g \beta B}^{\dagger} b_{h \gamma C}^{\dagger} b_{f_{2} \alpha_{2} A_{2}}^{\dagger}\left(T_{c}\right)_{A_{2} B_{2}}(\epsilon c)_{f_{2} \alpha_{2} A_{2}}^{\dagger}\left|0_{F}\right\rangle$
(f) $d_{a b c}\left(T_{F}\right)_{f g h}\left(\mathcal{E}_{A} T_{b}\right)_{B C}\left(\tau_{i} \epsilon\right)_{\alpha \beta} b_{f \alpha A}^{\dagger} b_{g \beta B}^{\dagger} b_{h \gamma C}^{\dagger} b_{f_{2} \alpha_{2} A_{2}}^{\dagger}\left(T_{c}\right)_{A_{2} B_{2}}(\epsilon c)_{f_{2} \alpha_{2} A_{2}}^{\dagger}\left|0_{F}\right\rangle$
(g) $f_{a b c}\left(T_{F}\right)_{f g h}\left(\mathcal{E}_{A} T_{b}\right)_{B C} \epsilon_{\alpha \beta} b_{f \alpha A}^{\dagger} b_{g \beta B}^{\dagger} b_{h \gamma C}^{\dagger} b_{f_{2} \alpha_{2} A_{2}}^{\dagger}\left(\tau_{i}\right)_{\alpha_{2} \beta_{2}}\left(T_{c}\right)_{A_{2} B_{2}}(\epsilon c)_{f_{2} \beta_{2} A_{2}}^{\dagger}\left|0_{F}\right\rangle$
(h) $d_{a b c}\left(T_{F}\right)_{f g h}\left(\mathcal{E}_{A} T_{b}\right)_{B C} \epsilon_{\alpha \beta} b_{f \alpha A}^{\dagger} b_{g \beta B}^{\dagger} b_{h \gamma C}^{\dagger} b_{f_{2} \alpha_{2} A_{2}}^{\dagger}\left(\tau_{i}\right)_{\alpha_{2} \beta_{2}}\left(T_{c}\right)_{A_{2} B_{2}}(\epsilon c)_{f_{2} \beta_{2} A_{2}}^{\dagger}\left|0_{F}\right\rangle$
(i) $\epsilon_{i j k}\left(T_{F}\right)_{f g h}\left(\mathcal{E}_{A}\right)_{B C}\left(\tau_{j} \epsilon\right)_{\alpha \beta} b_{f \alpha A}^{\dagger} b_{g \beta B}^{\dagger} b_{h \gamma C}^{\dagger} b_{f_{2} \alpha_{2} A_{2}}^{\dagger}\left(\tau_{k}\right)_{\alpha_{2} \beta_{2}}(\epsilon c)_{f_{2} \beta_{2} A_{2}}^{\dagger}\left|0_{F}\right\rangle$
(j) $\epsilon_{i j k}\left(T_{F}\right)_{f g h}\left(\mathcal{E}_{A}\right)_{B C}\left(\tau_{j} \epsilon\right)_{\alpha \beta} b_{f \alpha A}^{\dagger} b_{g \beta B}^{\dagger} b_{h \gamma C}^{\dagger} b_{f_{2} \alpha_{2} A_{2}}^{\dagger}\left(\tau_{k}\right)_{\alpha_{2} \beta_{2}}\left(T_{a}\right)_{A_{2} B_{2}}(\epsilon c)_{f_{2} \beta_{2} B_{2}}^{\dagger}\left|0_{F}\right\rangle$
(k) $\epsilon_{i j k} f_{a b c}\left(T_{F}\right)_{f g h}\left(\mathcal{E}_{A} T_{b}\right)_{B C}\left(\tau_{j} \epsilon\right)_{\alpha \beta} b_{f \alpha A}^{\dagger} b_{g \beta B}^{\dagger} b_{h \gamma C}^{\dagger} b_{f_{2} \alpha_{2} A_{2}}^{\dagger}\left(\tau_{k}\right)_{\alpha_{2} \beta_{2}}\left(T_{c}\right)_{A_{2} B_{2}}(\epsilon c)_{f_{2} \beta_{2} B_{2}}^{\dagger}\left|0_{F}\right\rangle$
(1) $\epsilon_{i j k} d_{a b c}\left(T_{F}\right)_{f g h}\left(\mathcal{E}_{A} T_{b}\right)_{B C}\left(\tau_{j} \epsilon\right)_{\alpha \beta} b_{f \alpha A}^{\dagger} b_{g \beta B}^{\dagger} b_{h \gamma C}^{\dagger} b_{f_{2} \alpha_{2} A_{2}}^{\dagger}\left(\tau_{k}\right)_{\alpha_{2} \beta_{2}}\left(T_{c}\right)_{A_{2} B_{2}}(\epsilon c)_{f_{2} \beta_{2} B_{2}}^{\dagger}\left|0_{F}\right\rangle$

(6) $\left(\mathbf{8}, \mathbf{2} \otimes \frac{1}{2}, \mathbf{8}\right)$ :
(a) $\left(T_{F}\right)_{f g h}\left(\mathcal{E}_{A} T_{a}\right)_{B C}\left(\tau_{i} \epsilon\right)_{\alpha \beta} b_{f \alpha A}^{\dagger} b_{g \beta B}^{\dagger} b_{h \gamma C}^{\dagger} b_{f_{2} \alpha_{2} A_{2}}^{\dagger}\left(\tau_{j}\right)_{\alpha_{2} \beta_{2}}(\epsilon c)_{f_{2} \beta_{2} A_{2}}^{\dagger}\left|0_{F}\right\rangle$
(b) $\left(T_{F}\right)_{f g h}\left(\mathcal{E}_{A}\right)_{B C}\left(\tau_{i} \epsilon\right)_{\alpha \beta} b_{f \alpha A}^{\dagger} b_{g \beta B}^{\dagger} b_{h \gamma C}^{\dagger} b_{f_{2} \alpha_{2} A_{2}}^{\dagger}\left(\tau_{j}\right)_{\alpha_{2} \beta_{2}}\left(T_{a}\right)_{A_{2} B_{2}}(\epsilon c)_{f_{2} \beta_{2} B_{2}}^{\dagger}\left|0_{F}\right\rangle$ 
(c) $f_{a b c}\left(T_{F}\right)_{f g h}\left(\mathcal{E}_{A} T_{b}\right)_{B C}\left(\tau_{i} \epsilon\right)_{\alpha \beta} b_{f \alpha A}^{\dagger} b_{g \beta B}^{\dagger} b_{h \gamma C}^{\dagger} b_{f_{2} \alpha_{2} A_{2}}^{\dagger}\left(\tau_{j}\right)_{\alpha_{2} \beta_{2}}\left(T_{c}\right)_{A_{2} B_{2}}(\epsilon c)_{f_{2} \beta_{2} A_{2}}^{\dagger}\left|0_{F}\right\rangle$

(d) $d_{a b c}\left(T_{F}\right)_{f g h}\left(\mathcal{E}_{A} T_{b}\right)_{B C}\left(\tau_{i} \epsilon\right)_{\alpha \beta} b_{f \alpha A}^{\dagger} b_{g \beta B}^{\dagger} b_{h \gamma C}^{\dagger} b_{f_{2} \alpha_{2} A_{2}}^{\dagger}\left(\tau_{j}\right)_{\alpha_{2} \beta_{2}}\left(T_{c}\right)_{A_{2} B_{2}}(\epsilon c)_{f_{2} \beta_{2} A_{2}}^{\dagger}\left|0_{F}\right\rangle$

(7) $\left(\mathbf{8}, \frac{1}{2}, 27 \oplus 20\right)$ :

(a) $\left(T_{F}\right)_{f g h}\left(\mathcal{E}_{A} T_{a}\right)_{B C} \epsilon_{\alpha \beta} b_{f \alpha A}^{\dagger} b_{g \beta B}^{\dagger} b_{h \gamma C}^{\dagger} b_{f_{2} \alpha_{2} A_{2}}^{\dagger}\left(T_{b}\right)_{A_{2} B_{2}}(\epsilon c)_{f_{2} \alpha_{2} B_{2}}^{\dagger}\left|0_{F}\right\rangle$

(b) $\left(T_{F}\right)_{f g h}\left(\mathcal{E}_{A} T_{a}\right)_{B C}\left(\tau_{i} \epsilon\right)_{\alpha \beta} b_{f \alpha A}^{\dagger} b_{g \beta B}^{\dagger} b_{h \gamma C}^{\dagger} b_{f_{2} \alpha_{2} A_{2}}^{\dagger}\left(\tau_{i}\right)_{\alpha_{2} \beta_{2}}\left(T_{b}\right)_{A_{1} B_{2}}(\epsilon c)_{f_{2} \beta_{2} B_{2}}^{\dagger}\left|0_{F}\right\rangle$

(8) $\left(\mathbf{8}, \mathbf{1} \otimes \frac{1}{2}, 27 \oplus \mathbf{2 0}\right)$ :

(a) $\left(T_{F}\right)_{f g h}\left(\mathcal{E}_{A} T_{a}\right)_{B C}\left(\tau_{i} \epsilon\right)_{\alpha \beta} b_{f \alpha A}^{\dagger} b_{g \beta B}^{\dagger} b_{h \gamma C}^{\dagger} b_{f_{2} \alpha_{2} A_{2}}^{\dagger}\left(T_{b}\right)_{A_{2} B_{2}}(\epsilon c)_{f_{2} \alpha_{2} B_{2}}^{\dagger}\left|0_{F}\right\rangle$

(b) $\left(T_{F}\right)_{f g h}\left(\mathcal{E}_{A} T_{a}\right)_{B C} \epsilon_{\alpha \beta} b_{f \alpha A}^{\dagger} b_{g \beta B}^{\dagger} b_{h \gamma C}^{\dagger} b_{f_{2} \alpha_{2} A_{2}}^{\dagger}\left(\tau_{i}\right)_{\alpha_{2} \beta_{2}}\left(T_{b}\right)_{A_{2} B_{2}}(\epsilon c)_{f_{2} \beta_{2} B_{2}}^{\dagger}\left|0_{F}\right\rangle$

(c) $\epsilon_{i j k}\left(T_{F}\right)_{f g h}\left(\mathcal{E}_{A} T_{a}\right)_{B C}\left(\tau_{j} \epsilon\right)_{\alpha \beta} b_{f \alpha A}^{\dagger} b_{g \beta B}^{\dagger} b_{h \gamma C}^{\dagger} b_{f_{2} \alpha_{2} A_{2}}^{\dagger}\left(\tau_{k}\right)_{\alpha_{2} \beta_{2}}\left(T_{b}\right)_{A_{1} B_{2}}(\epsilon c)_{f_{2} \beta_{2} B_{2}}^{\dagger}\left|0_{F}\right\rangle$

(9) $\left(\mathbf{8}, \mathbf{2} \otimes \frac{1}{2}, \mathbf{2 7} \oplus \mathbf{2 0}\right)$ :

(a) $\left(T_{F}\right)_{f g h}\left(\mathcal{E}_{A} T_{a}\right)_{B C}\left(\tau_{i} \epsilon\right)_{\alpha \beta} b_{f \alpha A}^{\dagger} b_{g \beta B}^{\dagger} b_{h \gamma C}^{\dagger} b_{f_{2} \alpha_{2} A_{2}}^{\dagger}\left(\tau_{j}\right)_{\alpha_{2} \beta_{2}}\left(T_{b}\right)_{A_{1} B_{2}}(\epsilon c)_{f_{2} \beta_{2} B_{2}}^{\dagger}\left|0_{F}\right\rangle$

(10) $\left(\mathbf{1 0}, \frac{1}{2}, \mathbf{1}\right)$ :

(a) $\Delta_{f g h}^{\rho}\left(\mathcal{E}_{A}\right)_{B C}\left(\tau_{i} \epsilon\right)_{\alpha \beta} b_{f \alpha A}^{\dagger} b_{g \beta B}^{\dagger} b_{h \gamma C}^{\dagger} b_{f_{2} \alpha_{2} A_{2}}^{\dagger}\left(\tau_{i}\right)_{\alpha_{2} \beta_{2}}(\epsilon c)_{f_{2} \beta_{2} A_{2}}^{\dagger}\left|0_{F}\right\rangle$

(b) $\Delta_{f g h}^{\rho}\left(\mathcal{E}_{A} T_{a}\right)_{B C} \epsilon_{\alpha \beta} b_{f \alpha A}^{\dagger} b_{g \beta B}^{\dagger} b_{h \gamma C}^{\dagger} b_{f_{2} \alpha_{2} A_{2}}^{\dagger}\left(T_{a}\right)_{A_{2} B_{2}}(\epsilon c)_{f_{2} \alpha_{2} A_{2}}^{\dagger}\left|0_{F}\right\rangle$

(c) $\Delta_{f g h}^{\rho}\left(\mathcal{E}_{A} T_{a}\right)_{B C}\left(\tau_{i} \epsilon\right)_{\alpha \beta} b_{f \alpha A}^{\dagger} b_{g \beta B}^{\dagger} b_{h \gamma C}^{\dagger} b_{f_{2} \alpha_{2} A_{2}}^{\dagger}\left(\tau_{i}\right)_{\alpha_{2} \beta_{2}}\left(T_{a}\right)_{A_{2} B_{2}}(\epsilon c)_{f_{2} \beta_{2} B_{2}}^{\dagger}\left|0_{F}\right\rangle$

(11) $\left(\mathbf{1 0}, \mathbf{1} \otimes \frac{1}{2}, \mathbf{1}\right):$

(a) $\Delta_{f g h}^{\rho}\left(\mathcal{E}_{A}\right)_{B C}\left(\tau_{i} \epsilon\right)_{\alpha \beta} b_{f \alpha A}^{\dagger} b_{g \beta B}^{\dagger} b_{h \gamma C}^{\dagger} b_{f_{2} \alpha_{2} A_{2}}^{\dagger}(\epsilon c)_{f_{2} \alpha_{2} A_{2}}^{\dagger}\left|0_{F}\right\rangle$

(b) $\Delta_{f g h}^{\rho}\left(\mathcal{E}_{A} T_{a}\right)_{B C} \epsilon_{\alpha \beta} b_{f \alpha A}^{\dagger} b_{g \beta B}^{\dagger} b_{h \gamma C}^{\dagger} b_{f_{2} \alpha_{2} A_{2}}^{\dagger}\left(\tau_{i}\right)_{\alpha_{2} \beta_{2}}\left(T_{a}\right)_{A_{2} B_{2}}(\epsilon c)_{f_{2} \beta_{2} B_{2}}^{\dagger}\left|0_{F}\right\rangle$

(c) $\Delta_{f g h}^{\rho}\left(\mathcal{E}_{A} T_{a}\right)_{B C}\left(\tau_{i} \epsilon\right)_{\alpha \beta} b_{f \alpha A}^{\dagger} b_{g \beta B}^{\dagger} b_{h \gamma C}^{\dagger} b_{f_{2} \alpha_{2} A_{2}}^{\dagger}\left(T_{a}\right)_{A_{2} B_{2}}(\epsilon c)_{f_{2} \alpha_{2} B_{2}}^{\dagger}\left|0_{F}\right\rangle$

(d) $\epsilon_{i j k} \Delta_{f g h}^{\rho}\left(\mathcal{E}_{A}\right)_{B C}\left(\tau_{j} \epsilon\right)_{\alpha \beta} b_{f \alpha A}^{\dagger} b_{g \beta B}^{\dagger} b_{h \gamma C}^{\dagger} b_{f_{2} \alpha_{2} A_{2}}^{\dagger}\left(\tau_{k}\right)_{\alpha_{2} \beta_{2}}(\epsilon c)_{f_{2} \beta_{2} A_{2}}^{\dagger}\left|0_{F}\right\rangle$

(e) $\epsilon_{i j k} \Delta_{f g h}^{\rho}\left(\mathcal{E}_{A} T_{a}\right)_{B C}\left(\tau_{j} \epsilon\right)_{\alpha \beta} b_{f \alpha A}^{\dagger} b_{g \beta B}^{\dagger} b_{h \gamma C}^{\dagger} b_{f_{2} \alpha_{2} A_{2}}^{\dagger}\left(\tau_{k}\right)_{\alpha_{2} \beta_{2}}\left(T_{a}\right)_{A_{2} B_{2}}(\epsilon c)_{f_{2} \beta_{2} B_{2}}^{\dagger}\left|0_{F}\right\rangle$

(12) $\left(\mathbf{1 0}, \mathbf{2} \otimes \frac{1}{2}, \mathbf{1}\right):$

(a) $\Delta_{f g h}^{\rho}\left(\mathcal{E}_{A}\right)_{B C}\left(\tau_{i} \epsilon\right)_{\alpha \beta} b_{f \alpha A}^{\dagger} b_{g \beta B}^{\dagger} b_{h \gamma C}^{\dagger} b_{f_{2} \alpha_{2} A_{2}}^{\dagger}\left(\tau_{j}\right)_{\alpha_{2} \beta_{2}}(\epsilon c)_{f_{2} \beta_{2} A_{2}}^{\dagger}\left|0_{F}\right\rangle$

(b) $\Delta_{f g h}^{\rho}\left(\mathcal{E}_{A} T_{a}\right)_{B C}\left(\tau_{i} \epsilon\right)_{\alpha \beta} b_{f \alpha A}^{\dagger} b_{g \beta B}^{\dagger} b_{h \gamma C}^{\dagger} b_{f_{2} \alpha_{2} A_{2}}^{\dagger}\left(\tau_{j}\right)_{\alpha_{2} \beta_{2}}\left(T_{a}\right)_{A_{2} B_{2}}(\epsilon c)_{f_{2} \beta_{2} A_{2}}^{\dagger}\left|0_{F}\right\rangle$

(13) $\left(\mathbf{1 0}, \frac{\mathbf{1}}{\mathbf{2}}, \mathbf{8}\right)$ :

(a) $\Delta_{f g h}^{\rho}\left(\mathcal{E}_{A} T_{a}\right)_{B C} \epsilon_{\alpha \beta} b_{f \alpha A}^{\dagger} b_{g \beta B}^{\dagger} b_{h \gamma C}^{\dagger} b_{f_{2} \alpha_{2} A_{2}}^{\dagger}(\epsilon c)_{f_{2} \alpha_{2} A_{2}}^{\dagger}\left|0_{F}\right\rangle$

(b) $\Delta_{f g h}^{\rho}\left(\mathcal{E}_{A}\right)_{B C}\left(\tau_{i} \epsilon\right)_{\alpha \beta} b_{f \alpha A}^{\dagger} b_{g \beta B}^{\dagger} b_{h \gamma C}^{\dagger} b_{f_{2} \alpha_{2} A_{2}}^{\dagger}\left(\tau_{i}\right)_{\alpha_{2} \beta_{2}}\left(T_{a}\right)_{A_{2} B_{2}}(\epsilon c)_{f_{2} \beta_{2} B_{2}}^{\dagger}\left|0_{F}\right\rangle$

(c) $\Delta_{f g h}^{\rho}\left(\mathcal{E}_{A} T_{a}\right)_{B C}\left(\tau_{i} \epsilon\right)_{\alpha \beta} b_{f \alpha A}^{\dagger} b_{g \beta B}^{\dagger} b_{h \gamma C}^{\dagger} b_{f_{2} \alpha_{2} A_{2}}^{\dagger}\left(\tau_{i}\right)_{\alpha_{2} \beta_{2}}(\epsilon c)_{f_{2} \beta_{2} A_{2}}^{\dagger}\left|0_{F}\right\rangle$

(d) $f_{a b c} \Delta_{f g h}^{\rho}\left(\mathcal{E}_{A} T_{b}\right)_{B C} \epsilon_{\alpha \beta} b_{f \alpha A}^{\dagger} b_{g \beta B}^{\dagger} b_{h \gamma C}^{\dagger} b_{f_{2} \alpha_{2} A_{2}}^{\dagger}\left(T_{c}\right)_{A_{2} B_{2}}(\epsilon c)_{f_{2} \alpha_{2} B_{2}}^{\dagger}\left|0_{F}\right\rangle$

(e) $d_{a b c} \Delta_{f g h}^{\rho}\left(\mathcal{E}_{A} T_{b}\right)_{B C} \epsilon_{\alpha \beta} b_{f \alpha A}^{\dagger} b_{g \beta B}^{\dagger} b_{h \gamma C}^{\dagger} b_{f_{2} \alpha_{2} A_{2}}^{\dagger}\left(T_{c}\right)_{A_{2} B_{2}}(\epsilon c)_{f_{2} \alpha_{2} B_{2}}^{\dagger}\left|0_{F}\right\rangle$

(f) $f_{a b c} \Delta_{f g h}^{\rho}\left(\mathcal{E}_{A} T_{b}\right)_{B C}\left(\tau_{i} \epsilon\right)_{\alpha \beta} b_{f \alpha A}^{\dagger} b_{g \beta B}^{\dagger} b_{h \gamma C}^{\dagger} b_{f_{2} \alpha_{2} A_{2}}^{\dagger}\left(\tau_{i}\right)_{\alpha_{2} \beta_{2}}\left(T_{c}\right)_{A_{2} B_{2}}(\epsilon c)_{f_{2} \beta_{2} B_{2}}^{\dagger}\left|0_{F}\right\rangle$

(g) $d_{a b c} \Delta_{f g h}^{\rho}\left(\mathcal{E}_{A} T_{b}\right)_{B C}\left(\tau_{i} \epsilon\right)_{\alpha \beta} b_{f \alpha A}^{\dagger} b_{g \beta B}^{\dagger} b_{h \gamma C}^{\dagger} b_{f_{2} \alpha_{2} A_{2}}^{\dagger}\left(\tau_{i}\right)_{\alpha_{2} \beta_{2}}\left(T_{c}\right)_{A_{2} B_{2}}(\epsilon c)_{f_{2} \beta_{2} B_{2}}^{\dagger}\left|0_{F}\right\rangle$

(14) $\left(\mathbf{1 0}, 1 \otimes \frac{1}{2}, \mathbf{8}\right)$ :

(a) $\Delta_{f g h}^{\rho}\left(\mathcal{E}_{A} T_{a}\right)_{B C}\left(\tau_{i} \epsilon\right)_{\alpha \beta} b_{f \alpha A}^{\dagger} b_{g \beta B}^{\dagger} b_{h \gamma C}^{\dagger} b_{f_{2} \alpha_{2} A_{2}}^{\dagger}(\epsilon c)_{f_{2} \alpha_{2} A_{2}}^{\dagger}\left|0_{F}\right\rangle$

(b) $\Delta_{f g h}^{\rho}\left(\mathcal{E}_{A}\right)_{B C}\left(\tau_{i} \epsilon\right)_{\alpha \beta} b_{f \alpha A}^{\dagger} b_{g \beta B}^{\dagger} b_{h \gamma C}^{\dagger} b_{f_{2} \alpha_{2} A_{2}}^{\dagger}\left(T_{a}\right)_{A_{2} B_{2}}(\epsilon c)_{f_{2} \alpha_{2} B_{2}}^{\dagger}\left|0_{F}\right\rangle$

(c) $\Delta_{f g h}^{\rho}\left(\mathcal{E}_{A} T_{a}\right)_{B C} \epsilon_{\alpha \beta} b_{f \alpha A}^{\dagger} b_{g \beta B}^{\dagger} b_{h \gamma C}^{\dagger} b_{f_{2} \alpha_{2} A_{2}}^{\dagger}\left(\tau_{i}\right)_{\alpha_{2} \beta_{2}}(\epsilon c)_{f_{2} \beta_{2} A_{2}}^{\dagger}\left|0_{F}\right\rangle$

(d) $f_{a b c} \Delta_{f g h}^{\rho}\left(\mathcal{E}_{A} T_{b}\right)_{B C}\left(\tau_{i} \epsilon\right)_{\alpha \beta} b_{f \alpha A}^{\dagger} b_{g \beta B}^{\dagger} b_{h \gamma C}^{\dagger} b_{f_{2} \alpha_{2} A_{2}}^{\dagger}\left(T_{c}\right)_{A_{2} B_{2}}(\epsilon c)_{f_{2} \alpha_{2} A_{2}}^{\dagger}\left|0_{F}\right\rangle$

(e) $d_{a b c} \Delta_{f g h}^{\rho}\left(\mathcal{E}_{A} T_{b}\right)_{B C}\left(\tau_{i} \epsilon\right)_{\alpha \beta} b_{f \alpha A}^{\dagger} b_{g \beta B}^{\dagger} b_{h \gamma C}^{\dagger} b_{f_{2} \alpha_{2} A_{2}}^{\dagger}\left(T_{c}\right)_{A_{2} B_{2}}(\epsilon c)_{f_{2} \alpha_{2} A_{2}}^{\dagger}\left|0_{F}\right\rangle$

(f) $f_{a b c} \Delta_{f g h}^{\rho}\left(\mathcal{E}_{A} T_{b}\right)_{B C} \epsilon_{\alpha \beta} b_{f \alpha A}^{\dagger} b_{g \beta B}^{\dagger} b_{h \gamma C}^{\dagger} b_{f_{2} \alpha_{2} A_{2}}^{\dagger}\left(\tau_{i}\right)_{\alpha_{2} \beta_{2}}\left(T_{c}\right)_{A_{2} B_{2}}(\epsilon c)_{f_{2} \beta_{2} A_{2}}^{\dagger}\left|0_{F}\right\rangle$

(g) $d_{a b c} \Delta_{f g h}^{\rho}\left(\mathcal{E}_{A} T_{b}\right)_{B C} \epsilon_{\alpha \beta} b_{f \alpha A}^{\dagger} b_{g \beta B}^{\dagger} b_{h \gamma C}^{\dagger} b_{f_{2} \alpha_{2} A_{2}}^{\dagger}\left(\tau_{i}\right)_{\alpha_{2} \beta_{2}}\left(T_{c}\right)_{A_{2} B_{2}}(\epsilon c)_{f_{2} \beta_{2} A_{2}}^{\dagger}\left|0_{F}\right\rangle$

(h) $\epsilon_{i j k} \Delta_{f g h}^{\rho}\left(\mathcal{E}_{A}\right)_{B C}\left(\tau_{j} \epsilon\right)_{\alpha \beta} b_{f \alpha A}^{\dagger} b_{g \beta B}^{\dagger} b_{h \gamma C}^{\dagger} b_{f_{2} \alpha_{2} A_{2}}^{\dagger}\left(\tau_{k}\right)_{\alpha_{2} \beta_{2}}(\epsilon c)_{f_{2} \beta_{2} A_{2}}^{\dagger}\left|0_{F}\right\rangle$

(i) $\epsilon_{i j k} \Delta_{f g h}^{\rho}\left(\mathcal{E}_{A}\right)_{B C}\left(\tau_{j} \epsilon\right)_{\alpha \beta} b_{f \alpha A}^{\dagger} b_{g \beta B}^{\dagger} b_{h \gamma C}^{\dagger} b_{f_{2} \alpha_{2} A_{2}}^{\dagger}\left(\tau_{k}\right)_{\alpha_{2} \beta_{2}}\left(T_{a}\right)_{A_{2} B_{2}}(\epsilon c)_{f_{2} \beta_{2} B_{2}}^{\dagger}\left|0_{F}\right\rangle$

(j) $\epsilon_{i j k} f_{a b c} \Delta_{f g h}^{\rho}\left(\mathcal{E}_{A} T_{b}\right)_{B C}\left(\tau_{j} \epsilon\right)_{\alpha \beta} b_{f \alpha A}^{\dagger} b_{g \beta B}^{\dagger} b_{h \gamma C}^{\dagger} b_{f_{2} \alpha_{2} A_{2}}^{\dagger}\left(\tau_{k}\right)_{\alpha_{2} \beta_{2}}\left(T_{c}\right)_{A_{2} B_{2}}(\epsilon c)_{f_{2} \beta_{2} B_{2}}^{\dagger}\left|0_{F}\right\rangle$

(k) $\epsilon_{i j k} d_{a b c} \Delta_{f g h}^{\rho}\left(\mathcal{E}_{A} T_{b}\right)_{B C}\left(\tau_{j} \epsilon\right)_{\alpha \beta} b_{f \alpha A}^{\dagger} b_{g \beta B}^{\dagger} b_{h \gamma C}^{\dagger} b_{f_{2} \alpha_{2} A_{2}}^{\dagger}\left(\tau_{k}\right)_{\alpha_{2} \beta_{2}}\left(T_{c}\right)_{A_{2} B_{2}}(\epsilon c)_{f_{2} \beta_{2} B_{2}}^{\dagger}\left|0_{F}\right\rangle$ 
(15) $\left(\mathbf{1 0}, 2 \otimes \frac{1}{2}, \mathbf{8}\right):$

(a) $\Delta_{f g h}^{\rho}\left(\mathcal{E}_{A} T_{a}\right)_{B C}\left(\tau_{i} \epsilon\right)_{\alpha \beta} b_{f \alpha A}^{\dagger} b_{g \beta B}^{\dagger} b_{h \gamma C}^{\dagger} b_{f_{2} \alpha_{2} A_{2}}^{\dagger}\left(\tau_{j}\right)_{\alpha_{2} \beta_{2}}(\epsilon c)_{f_{2} \beta_{2} A_{2}}^{\dagger}\left|0_{F}\right\rangle$

(b) $\Delta_{f g h}^{\gamma^{\prime}}\left(\mathcal{E}_{A}\right)_{B C}\left(\tau_{i} \epsilon\right)_{\alpha \beta} b_{f \alpha A}^{\dagger} b_{g \beta B}^{\dagger} b_{h \gamma C}^{\dagger} b_{f_{2} \alpha_{2} A_{2}}^{\dagger}\left(\tau_{j}\right)_{\alpha_{2} \beta_{2}}\left(T_{a}\right)_{A_{2} B_{2}}(\epsilon c)_{f_{2} \beta_{2} B_{2}}^{\dagger}\left|0_{F}\right\rangle$

(c) $f_{a b c} \Delta_{f g h}^{\rho}\left(\mathcal{E}_{A} T_{b}\right)_{B C}\left(\tau_{i} \epsilon\right)_{\alpha \beta} b_{f \alpha A}^{\dagger} b_{g \beta B}^{\dagger} b_{h \gamma C}^{\dagger} b_{f_{2} \alpha_{2} A_{2}}^{\dagger}\left(\tau_{j}\right)_{\alpha_{2} \beta_{2}}\left(T_{c}\right)_{A_{2} B_{2}}(\epsilon c)_{f_{2} \beta_{2} A_{2}}^{\dagger}\left|0_{F}\right\rangle$

(d) $d_{a b c} \Delta_{f g h}^{\rho}\left(\mathcal{E}_{A} T_{b}\right)_{B C}\left(\tau_{i} \epsilon\right)_{\alpha \beta} b_{f \alpha A}^{\dagger} b_{g \beta B}^{\dagger} b_{h \gamma C}^{\dagger} b_{f_{2} \alpha_{2} A_{2}}^{\dagger}\left(\tau_{j}\right)_{\alpha_{2} \beta_{2}}\left(T_{c}\right)_{A_{2} B_{2}}(\epsilon c)_{f_{2} \beta_{2} A_{2}}^{\dagger}\left|0_{F}\right\rangle$

(16) $\left(\mathbf{1 0}, \frac{1}{2}, 27 \oplus 20\right)$ :

(a) $\Delta_{f g h}^{\rho}\left(\mathcal{E}_{A} T_{a}\right)_{B C} \epsilon_{\alpha \beta} b_{f \alpha A}^{\dagger} b_{g \beta B}^{\dagger} b_{h \gamma C}^{\dagger} b_{f_{2} \alpha_{2} A_{2}}^{\dagger}\left(T_{b}\right)_{A_{2} B_{2}}(\epsilon c)_{f_{2} \alpha_{2} B_{2}}^{\dagger}\left|0_{F}\right\rangle$

(b) $\Delta_{f g h}^{\rho}\left(\mathcal{E}_{A} T_{a}\right)_{B C}\left(\tau_{i} \epsilon\right)_{\alpha \beta} b_{f \alpha A}^{\dagger} b_{g \beta B}^{\dagger} b_{h \gamma C}^{\dagger} b_{f_{2} \alpha_{2} A_{2}}^{\dagger}\left(\tau_{i}\right)_{\alpha_{2} \beta_{2}}\left(T_{b}\right)_{A_{1} B_{2}}(\epsilon c)_{f_{2} \beta_{2} B_{2}}^{\dagger}\left|0_{F}\right\rangle$

(17) $\left(\mathbf{1 0}, 1 \otimes \frac{1}{2}, 27 \oplus \mathbf{2 0}\right)$ :

(a) $\Delta_{f g h}^{\rho}\left(\mathcal{E}_{A} T_{a}\right)_{B C}\left(\tau_{i} \epsilon\right)_{\alpha \beta} b_{f \alpha A}^{\dagger} b_{g \beta B}^{\dagger} b_{h \gamma C}^{\dagger} b_{f_{2} \alpha_{2} A_{2}}^{\dagger}\left(T_{b}\right)_{A_{2} B_{2}}(\epsilon c)_{f_{2} \alpha_{2} B_{2}}^{\dagger}\left|0_{F}\right\rangle$

(b) $\Delta_{f g h}^{\rho}\left(\mathcal{E}_{A} T_{a}\right)_{B C} \epsilon_{\alpha \beta} b_{f \alpha A}^{\dagger} b_{g \beta B}^{\dagger} b_{h \gamma C}^{\dagger} b_{f_{2} \alpha_{2} A_{2}}^{\dagger}\left(\tau_{i}\right)_{\alpha_{2} \beta_{2}}\left(T_{b}\right)_{A_{2} B_{2}}(\epsilon c)_{f_{2} \beta_{2} B_{2}}^{\dagger}\left|0_{F}\right\rangle$

(c) $\epsilon_{i j k} \Delta_{f g h}^{\rho}\left(\mathcal{E}_{A} T_{a}\right)_{B C}\left(\tau_{j} \epsilon\right)_{\alpha \beta} b_{f \alpha A}^{\dagger} b_{g \beta B}^{\dagger} b_{h \gamma C}^{\dagger} b_{f_{2} \alpha_{2} A_{2}}^{\dagger}\left(\tau_{k}\right)_{\alpha_{2} \beta_{2}}\left(T_{b}\right)_{A_{1} B_{2}}(\epsilon c)_{f_{2} \beta_{2} B_{2}}^{\dagger}\left|0_{F}\right\rangle$

(18) $\left(10,2 \otimes \frac{1}{2}, 27 \oplus 20\right)$ :

(a) $\Delta_{f g h}^{\rho}\left(\mathcal{E}_{A} T_{a}\right)_{B C}\left(\tau_{i} \epsilon\right)_{\alpha \beta} b_{f \alpha A}^{\dagger} b_{g \beta B}^{\dagger} b_{h \gamma C}^{\dagger} b_{f_{2} \alpha_{2} A_{2}}^{\dagger}\left(\tau_{j}\right)_{\alpha_{2} \beta_{2}}\left(T_{b}\right)_{A_{1} B_{2}}(\epsilon c)_{f_{2} \beta_{2} B_{2}}^{\dagger}\left|0_{F}\right\rangle$

\section{GLUON STATES}

As gluon trial wave functions, we choose eigenstates of a 24-dimensional harmonic oscillator, defined in (3.6). Introducing oscillator creation and annihilation operators,

$$
\begin{aligned}
& A_{i a}=\frac{1}{\sqrt{2}}\left(i \Pi_{i a}+M_{i a}\right), \\
& A_{i a}^{\dagger}=\frac{1}{\sqrt{2}}\left(-i \Pi_{i a}+M_{i a}\right),
\end{aligned}
$$

we create oscillator eigenstates by successive operations of $A^{\dagger}$ on the oscillator vacuum $\left|0_{B}\right\rangle$. These states can be organized into real representations of $\operatorname{Ad} S U(3)$, formed by decomposing tensor products of the adjoint representation.

To write gluon states, we define a matrix $B$ whose elements are oscillator creation operators.

$$
B_{i a}=A_{i a}^{\dagger} .
$$

A gluon state with a given number of oscillators can be expressed as a function of $B$ acting on the oscillator vacuum $\left|0_{B}\right\rangle$. We define $\left(D_{a}\right)_{b c} \equiv d_{a b c}$ and $\left(F_{a}\right)_{b c} \equiv f_{a b c}$. The gluon states are organized according to their spin and color. To make variational estimates of spin- 1 mesons and spin- $\frac{3}{2}$ baryons, we need the gluon states to have spin up to 3 . Again, for notational convenience, a spin-3 state is written with three free adjoint indices $i, j, k$, and the spin-3 state is obtained from a state $\left|\phi_{i j k}\right\rangle$ as

$\left|\phi_{i j k}^{(3)}\right\rangle=\left|\phi_{(i j k)}\right\rangle-\frac{2}{5} \sum_{l} \delta_{(i j}\left[\left|\phi_{k) l l}\right\rangle+\left|\phi_{l k) l}\right\rangle+\left|\phi_{l l k)}\right\rangle\right]$

where the brackets around the indices $(i j k)$ denote symmetrization in $i, j, k$.

\section{a. Gluon states}

(1) $(\mathbf{0}, \mathbf{1})$ :

(a) $\left|0_{B}\right\rangle$

(b) $\left(\operatorname{Tr} B B^{T}\right)\left|0_{B}\right\rangle$

(c) $\epsilon_{i j k} f_{a b c} B_{i a} B_{j b} B_{k c}\left|0_{B}\right\rangle$

(d) $\left(\operatorname{Tr} B B^{T}\right)^{2}\left|0_{B}\right\rangle$

(e) $\left(\operatorname{Tr} B B^{T} B B^{T}\right)\left|0_{B}\right\rangle$

(f) $\left(\operatorname{Tr} B D_{a} B^{T}\right)\left(\operatorname{Tr} B D_{a} B^{T}\right)\left|0_{B}\right\rangle$

(2) $(\mathbf{1}, \mathbf{1})$ :

(a) $B_{i a}\left(\operatorname{Tr} B D_{a} B^{T}\right)\left|0_{B}\right\rangle$

(b) $\epsilon_{j k l}\left(B D_{c} B^{T}\right)_{i j}\left(B F_{c} B^{T}\right)_{k l}\left|0_{B}\right\rangle$

(3) $(\mathbf{2}, \mathbf{1})$ :

(a) $\left(B B^{T}\right)_{i j}\left|0_{B}\right\rangle$

(b) $\left(\operatorname{Tr} B B^{T}\right)\left(B B^{T}\right)_{i j}\left|0_{B}\right\rangle$

(c) $\left(B B^{T} B B^{T}\right)_{i j}\left|0_{B}\right\rangle$

(d) $\left(\operatorname{Tr} B D_{a} B^{T}\right)\left(B D_{a} B^{T}\right)_{i j}\left|0_{B}\right\rangle$

(4) $(\mathbf{0 ,}, \mathbf{8})$ :

(a) $\left(\operatorname{Tr} B D_{a} B^{T}\right)\left|0_{B}\right\rangle$

(b) $\epsilon_{i j k}\left(B F_{c} B^{T}\right)_{i j}\left(B D_{c}\right)_{k a}\left|0_{B}\right\rangle$

(c) $\left(\operatorname{Tr} B B^{T}\right)\left(\operatorname{Tr} B D_{a} B^{T}\right)\left|0_{B}\right\rangle$

(d) $\left(\operatorname{Tr} B^{T} B D_{a} B^{T} B\right)\left|0_{B}\right\rangle$

(e) $\left(\operatorname{Tr} B D_{b} B^{T}\right)\left(B^{T} B\right)_{a b}\left|0_{B}\right\rangle$

(5) $(\mathbf{1}, \mathbf{8})$ :

(f) $\left(\operatorname{Tr} B D_{b} D_{a} B^{T}\right)\left(\operatorname{Tr} B D_{b} B^{T}\right)\left|0_{B}\right\rangle$

(a) $B_{i a}\left|0_{B}\right\rangle$

(b) $\epsilon_{i j k} f_{a b c} B_{j b} B_{k c}\left|0_{B}\right\rangle$

(c) $B_{i a}\left(\operatorname{Tr} B B^{T}\right)\left|0_{B}\right\rangle$

(d) $\left(B B^{T} B\right)_{i a}\left|0_{B}\right\rangle$

(e) $\operatorname{Tr}\left(B D_{c} B^{T}\right)\left(B D_{c}\right)_{i a}\left|0_{B}\right\rangle$

(f) $\operatorname{Tr}\left(B D_{c} B^{T}\right)\left(B F_{c}\right)_{i a}\left|0_{B}\right\rangle$

(g) $\epsilon_{j k l} f_{b c d} B_{j b} B_{k c} B_{l d} B_{i a}\left|0_{B}\right\rangle$

(h) $\epsilon_{j k l}\left(B B^{T}\right)_{i j}\left(B F_{a} B^{T}\right)_{k l}\left|0_{B}\right\rangle$

(i) $\epsilon_{j k l}\left(B D_{a} D_{b} B^{T}\right)_{i j}\left(B F_{b} B^{T}\right)_{k l}\left|0_{B}\right\rangle$

(j) $\epsilon_{j k l}\left(B F_{a} D_{b} B^{T}\right)_{i j}\left(B F_{b} B^{T}\right)_{k l}\left|0_{B}\right\rangle$

(k) $\epsilon_{i j k}\left(B F_{a} B^{T}\right)_{j k}\left(\operatorname{Tr}\left(B B^{T}\right)\left|0_{B}\right\rangle\right.$ 
(l) $\epsilon_{i j k}\left(\operatorname{Tr} B D_{b} B^{T}\right) B_{j c} B_{k a}\left|0_{B}\right\rangle$

(m) $\epsilon_{i j k}\left(\operatorname{Tr} B D_{b} B^{T}\right)\left(B F_{b} D_{a} B^{T}\right)_{j k}\left|0_{B}\right\rangle$

(6) $(\mathbf{2}, \mathbf{8})$ :

(n) $\epsilon_{i j k}\left(\operatorname{Tr} B D_{b} B^{T}\right)\left(B F_{b} F_{a} B^{T}\right)_{j k}\left|0_{B}\right\rangle$
(a) $\left(B D_{a} B^{T}\right)_{i j}\left|0_{B}\right\rangle$
(b) $\epsilon_{i l m}\left(B B^{T}\right)_{j l} B_{m a}\left|0_{B}\right\rangle$
(c) $\epsilon_{i l m}\left(B F_{c} B^{T}\right)_{j l}\left(B D_{c}\right)_{m a}\left|0_{B}\right\rangle$
(d) $\epsilon_{i l m}\left(B F_{c} B^{T}\right)_{j l}\left(B F_{c}\right)_{m a}\left|0_{B}\right\rangle$
(e) $\left(\operatorname{Tr} B D_{b} B^{T}\right) B_{i a} B_{j b}\left|0_{B}\right\rangle$
(f) $\left(B B^{T}\right)_{i j}\left(\operatorname{Tr} B D_{a} B^{T}\right)\left|0_{B}\right\rangle$
(g) $\left(\operatorname{Tr} B B^{T}\right)\left(B D_{a} B^{T}\right)_{i j}\left|0_{B}\right\rangle$
(h) $\left(B B^{T} B D_{a} B^{T}\right)_{i j}\left|0_{B}\right\rangle$
(i) $\left(B B^{T} B F_{a} B^{T}\right)_{i j}\left|0_{B}\right\rangle$
(j) $\left(\operatorname{Tr} B F_{b} F_{a} B^{T}\right)\left(B D_{b} B^{T}\right)_{i j}\left|0_{B}\right\rangle$
(k) $\left(\operatorname{Tr} B D_{b} D_{a} B^{T}\right)\left(B F_{b} B^{T}\right)_{i j}\left|0_{B}\right\rangle$
(l) $\left(\operatorname{Tr} B D_{b} F_{a} B^{T}\right)\left(B F_{b} B^{T}\right)_{i j}\left|0_{B}\right\rangle$
(m) $\left(\operatorname{Tr} B D_{b} B^{T}\right)\left(B D_{b} D_{a} B^{T}\right)_{i j}\left|0_{B}\right\rangle$
(n) $\left(\operatorname{Tr} B D_{b} B^{T}\right)\left(B D_{b} F_{a} B^{T}\right)_{i j}\left|0_{B}\right\rangle$
(o) $\left(\operatorname{Tr} B D_{b} B^{T}\right)\left(B F_{b} D_{a} B^{T}\right)_{i j}\left|0_{B}\right\rangle$
(p) $\left(\operatorname{Tr} B D_{b} B^{T}\right)\left(B F_{b} F_{a} B^{T}\right)_{i j}\left|0_{B}\right\rangle$

(7) $(\mathbf{0 , 2 7} \oplus \mathbf{2 0})$ :
(a) $\left(B^{T} B\right)_{a b}\left|0_{B}\right\rangle$
(b) $\epsilon_{i j k}\left(B F_{a} B^{T}\right)_{i j} B_{k b}\left|0_{B}\right\rangle$
(c) $\left(\operatorname{Tr} B^{T} B\right)\left(B^{T} B\right)_{a b}\left|0_{B}\right\rangle$
(d) $\left(B^{T} B B^{T} B\right)_{a b}\left|0_{B}\right\rangle$
(e) $\left(\operatorname{Tr} B^{T} B D_{c}\right)\left(B^{T} B D_{c}\right)_{a b}\left|0_{B}\right\rangle$
(f) $\left(\operatorname{Tr} B^{T} B D_{c}\right)\left(B^{T} B F_{c}\right)_{a b}\left|0_{B}\right\rangle$

(8) $(\mathbf{1}, 27 \oplus \mathbf{2 0})$ :
(a) $\epsilon_{i j k} B_{j a} B_{k b}\left|0_{B}\right\rangle$
(b) $\left(\operatorname{Tr} B D_{a} B^{T}\right) B_{i b}\left|0_{B}\right\rangle$
(c) $\left(B F_{a} B^{T} B\right)_{i b}\left|0_{B}\right\rangle$
(d) $\left(B D_{a} B^{T} B\right)_{i b}\left|0_{B}\right\rangle$

(e) $\epsilon_{i_{1} j_{1} k_{1}}\left(B F_{c} B^{T}\right)_{i_{1} j_{1}}\left(B D_{c}\right)_{k_{1} a} B_{i b}\left|0_{B}\right\rangle$

(f) $\epsilon_{i j k}\left(\operatorname{Tr} B B^{T}\right) B_{j a} B_{k b}\left|0_{B}\right\rangle$

(g) $\epsilon_{i j k} B_{j a}\left(B B^{T} B\right)_{k b}\left|0_{B}\right\rangle$

(h) $\epsilon_{i j k}\left(\operatorname{Tr} B D_{c} B^{T}\right)\left(B D_{c}\right)_{j a} B_{k b}\left|0_{B}\right\rangle$

(i) $\epsilon_{i j k}\left(B D_{c}\right)_{j a}\left(B D_{c} B^{T} B\right)_{k b}\left|0_{B}\right\rangle$

(j) $\epsilon_{i j k}\left(\operatorname{Tr} B D_{c} B^{T}\right)\left(B F_{c}\right)_{j a} B_{k b}\left|0_{B}\right\rangle$

(k) $\epsilon_{i j k}\left(B F_{c} B^{T}\right)_{j k}\left(B^{T} B D_{c}\right)_{a b}\left|0_{B}\right\rangle$

(9) $(\mathbf{2}, \mathbf{2 7} \oplus \mathbf{2 0})$ :

(a) $B_{i a} B_{j b}\left|0_{B}\right\rangle$

(b) $\epsilon_{i l m}\left(B F_{a} B^{T}\right)_{l m} B_{j b}\left|0_{B}\right\rangle$

(c) $\epsilon_{i l m}\left(B D_{a} B^{T}\right)_{l m} B_{j b}\left|0_{B}\right\rangle$

(d) $\left(\operatorname{Tr} B B^{T}\right) B_{i a} B_{j b}\left|0_{B}\right\rangle$

(e) $\left(B B^{T}\right)_{i j}\left(B^{T} B\right)_{a b}\left|0_{B}\right\rangle$

(f) $\left(B B^{T} B\right)_{i a} B_{j b}\left|0_{B}\right\rangle$

(g) $\left(\operatorname{Tr} B D_{c} B^{T}\right)\left(B D_{c}\right)_{i a} B_{j b}\left|0_{B}\right\rangle$

(h) $\left(B D_{c} B^{T}\right)_{i j}\left(B^{T} B D_{c}\right)_{a b}\left|0_{B}\right\rangle$

(i) $\left(\operatorname{Tr} B D_{c} B^{T}\right)\left(B F_{c}\right)_{i a} B_{j b}\left|0_{B}\right\rangle$

(j) $\left(B D_{c} B^{T} B\right)_{i a}\left(B F_{c}\right)_{j b}\left|0_{B}\right\rangle$

(k) $\left(B F_{c} B^{T}\right)_{i j}\left(B D_{c} B^{T}\right)_{a b}\left|0_{B}\right\rangle$

(10) $(\mathbf{3}, \mathbf{1})$ :

(a) $d_{a b c} B_{i a} B_{j b} B_{k c}\left|0_{B}\right\rangle$

(11) $(\mathbf{3}, \mathbf{8})$ :

(a) $\left(B B^{T}\right)_{i j} B_{k a}\left|0_{B}\right\rangle$

(b) $\epsilon_{i l m}\left(B B^{T}\right)_{j l}\left(B D_{a} B^{T}\right)_{k m}\left|0_{B}\right\rangle$

(c) $\epsilon_{i l m}\left(B B^{T}\right)_{j k}\left(B F_{a} B^{T}\right)_{j m}\left|0_{B}\right\rangle$

(d) $\epsilon_{i l m}\left(B D_{b} B^{T}\right)_{j k}\left(B D_{b} D_{a} B^{T}\right)_{l m}\left|0_{B}\right\rangle$

(e) $\epsilon_{i l m}\left(B D_{b} B^{T}\right)_{j k}\left(B D_{b} F_{a} B^{T}\right)_{j m}\left|0_{B}\right\rangle$

(12) $(\mathbf{3}, 27 \oplus \mathbf{2 0})$ :
(a) $\left(B D_{a} B^{T}\right)_{i j} B_{k c}\left|0_{B}\right\rangle$
(b) $\epsilon_{i l m}\left(B B^{T}\right)_{j l} B_{k a} B_{m b}\left|0_{B}\right\rangle$
(c) $\epsilon_{i l m}\left(B D_{b} B^{T}\right)_{j l}\left(B D_{b}\right)_{k a} B_{m b}\left|0_{B}\right\rangle$
(d) $\epsilon_{i l m}\left(B D_{b} B^{T}\right)_{j l}\left(B F_{b}\right)_{k a} B_{m b}\left|0_{B}\right\rangle$

[1] S. Durr et al., Science 322, 1224 (2008).

[2] S. Durr et al., Phys. Rev. D 79, 014501 (2009).

[3] C. T. H. Davies et al., Phys. Rev. Lett. 92, 022001 (2004).

[4] S. Aoki et al., Phys. Rev. D 79, 034503 (2009).

[5] C. W. Bernard et al., Phys. Rev. D 64, 054506 (2001).

[6] C. Aubin, C. Bernard, C. DeTar, J. Osborn, S. Gottlieb, E. B. Gregory, D. Toussaint, U. M. Heller, J. E. Hetrick, and R. Sugar, Phys. Rev. D 70, 094505 (2004).

[7] N. Ukita et al., Proc. Sci., LAT2007 (2007) 138 [arXiv: 0710.3462].

[8] D. J. Antonio et al., Phys. Rev. D 75, 114501 (2007).

[9] C. Alexandrou et al., Phys. Rev. D 78, 014509 (2008).

[10] C. Alexandrou and C. Kallidonis, Phys. Rev. D 96, 034511 (2017).

[11] C. Alexandrou, V. Drach, K. Jansen, C. Kallidonis, and G. Koutsou, Phys. Rev. D 90, 074501 (2014).

[12] W. Bietenholz et al., Phys. Rev. D 84, 054509 (2011).
[13] J. Noaki et al. (JLQCD Collaboration), Proc. Sci., LAT2007 (2007) 126 [arXiv:0710.0929].

[14] S. Borsanyi et al., Science 347, 1452 (2015).

[15] K. Jansen, C. McNeile, C. Michael, and C. Urbach (ETM Collaboration), Phys. Rev. D 80, 054510 (2009).

[16] S. Aoki et al. (CS Collaboration), Phys. Rev. D 84, 094505 (2011).

[17] T. Rae, S. Collins, S. Durr, and S. Hofmann, Proc. Sci., LATTICE2016 (2017) 367.

[18] G. Bali, S. Collins, and P. Perez-Rubio, J. Phys. Conf. Ser. 426, 012017 (2013).

[19] P. Prez-Rubio, S. Collins, and G. S. Bali, Phys. Rev. D 92, 034504 (2015).

[20] S. Basak, S. Datta, M. Padmanath, P. Majumdar, and N. Mathur, Proc. Sci., LATTICE2012 (2012) 141 [arXiv:1211.6277].

[21] S. Basak, S. Datta, A. T. Lytle, M. Padmanath, P. Majumdar, and N. Mathur, Proc. Sci., LATTICE2013 (2014) 243 [arXiv:1312.3050]. 
[22] S. Basak et al. (ILGTI Collaboration), Proc. Sci., LATTICE2014 (2015) 083 [arXiv:1412.7248].

[23] N. Mathur and M. Padmanath, Phys. Rev. D 99, 031501 (2019).

[24] R. A. Briceno, H. W. Lin, and D. R. Bolton, Phys. Rev. D 86, 094504 (2012).

[25] Y. C. Chen, and T.-W. Chiu (TWQCD Collaboration), Phys. Lett. B 767, 193 (2017).

[26] A. P. Balachandran, S. Vaidya, and A. R. de Queiroz, Mod. Phys. Lett. A 30, 1550080 (2015).

[27] A. P. Balachandran, A. de Queiroz, and S. Vaidya, Int. J. Mod. Phys. A 30, 1550064 (2015).

[28] N. Acharyya, A. P. Balachandran, M. Pandey, S. Sanyal, and S. Vaidya, Int. J. Mod. Phys. A 33, 1850073 (2018).

[29] R. C. Myers, J. High Energy Phys. 12 (1999) 022.
[30] D. Sen, J. Math. Phys. (N.Y.) 27, 472 (1986).

[31] M. Tanabashi et al. (Particle Data Group), Phys. Rev. D 98, 030001 (2018).

[32] T. N. Pham, Phys. Rev. D 92, 054021 (2015).

[33] S. Weinberg, Phys. Rev. D 11, 3583 (1975).

[34] E. Witten, Nucl. Phys. B156, 269 (1979).

[35] G. Veneziano, Phys. Lett. 95B, 90 (1980).

[36] T. Feldmann, P. Kroll, and B. Stech, Phys. Rev. D 58, 114006 (1998).

[37] S. D. Bass and P. Moskal, Rev. Mod. Phys. 91, 015003 (2019).

[38] R. L. Jaffe, Nucl. Phys. B, Proc. Suppl. 142, 343 (2005).

[39] R. L. Jaffe, Phys. Rev. D 15, 267 (1977).

[40] A. Chodos, R. L. Jaffe, K. Johnson, and C. B. Thorn, Phys. Rev. D 10, 2599 (1974).

[41] T. A. DeGrand, R. L. Jaffe, K. Johnson, and J. E. Kiskis, Phys. Rev. D 12, 2060 (1975). 